\title{
Phosphorylation by the stress-activated MAPK Slt2 down-regulates the yeast TOR complex 2
}

\author{
Kristin L. Leskoske, ${ }^{1,3,6}$ Françoise M. Roelants, ${ }^{1,6}$ Anita Emmerstorfer-Augustin, ${ }^{1,4}$ \\ Christoph M. Augustin, ${ }^{2,5}$ Edward P. Si, ${ }^{1}$ Jennifer M. Hill, ${ }^{1}$ and Jeremy Thorner ${ }^{1}$ \\ ${ }^{1}$ Department of Molecular and Cell Biology, ${ }^{2}$ Department of Mechanical Engineering, University of California at Berkeley, \\ Berkeley, California 94720, USA
}

Saccharomyces cerevisiae target of rapamycin (TOR) complex 2 (TORC2) is an essential regulator of plasma membrane lipid and protein homeostasis. How TORC2 activity is modulated in response to changes in the status of the cell envelope is unclear. Here we document that TORC2 subunit Avo2 is a direct target of Slt2, the mitogenactivated protein kinase (MAPK) of the cell wall integrity pathway. Activation of Slt2 by overexpression of a constitutively active allele of an upstream Slt2 activator (Pkc1) or by auxin-induced degradation of a negative Slt2 regulator (Sln1) caused hyperphosphorylation of Avo2 at its MAPK phosphoacceptor sites in a Slt2-dependent manner and diminished TORC2-mediated phosphorylation of its major downstream effector, protein kinase Ypk1. Deletion of Avo2 or expression of a phosphomimetic Avo2 allele rendered cells sensitive to two stresses (myriocin treatment and elevated exogenous acetic acid) that the cell requires Ypk1 activation by TORC2 to survive. Thus, Avo2 is necessary for optimal TORC2 activity, and Slt2-mediated phosphorylation of Avo2 down-regulates TORC2 signaling. Compared with wild-type Avo2, phosphomimetic Avo2 shows significant displacement from the plasma membrane, suggesting that Slt2 inhibits TORC2 by promoting Avo2 dissociation. Our findings are the first demonstration that TORC2 function is regulated by MAPK-mediated phosphorylation.

[Keywords: signal transduction; protein kinase; regulation; yeast; mutants]

Supplemental material is available for this article.

Received July 6, 2018; revised version accepted October 10, 2018.

Cellular homeostasis requires careful coordination of metabolic growth processes and adaptive stress responses. Multiple signaling networks coordinate expansion at the cell boundary with the accumulation of intracellular mass and progression through the cell cycle and also play critical roles in responding to environmental fluctuations that compromise the integrity of the plasma membrane (PM) and, in yeast, the cell wall (Levin 2011).

Like all eukaryotes, yeast contains two evolutionarily conserved multicomponent protein kinase complexes in which a TOR (target of rapamycin) polypeptide is the catalytic subunit: TOR complex 1 (TORC1) and TORC2. TORC1 and TORC2 are essential regulators of growth and homeostasis but control different aspects of yeast

Present addresses: ${ }^{3}$ Collaborative Center for Translational Mass Spectrometry, Translational Genomics Research Institute, Phoenix, AZ 85004, USA; ${ }^{4}$ Institute of Molecular Biotechnology, Graz University of Technology, Graz 8010, Austria; ${ }^{5}$ Institute of Biophysics, Medical University of Graz, Graz 8010, Austria.

${ }^{6}$ These authors contributed equally to this work.

Corresponding author: jthorner@berkeley.edu

Article published online ahead of print. Article and publication date are online at http://www.genesdev.org/cgi/doi/10.1101/gad.318709.118. Freely available online through the Genes \& Development Open Access option. cell physiology (González and Hall 2017; Tatebe and Shiozaki 2017). TORC2 itself localizes to the PM (Berchtold and Walther 2009). TORC2 is thought to monitor the condition of the cell envelope (PM and cell wall) and control, through downstream effectors, processes that maintain the integrity and function of these vital barriers. The primary target of TORC2 is the AGC family protein kinase Ypk1 (and its paralog, Ypk2) (Kamada et al. 2005; Roelants et al. 2011; Berchtold et al. 2012; Niles et al. 2012). TORC2 activates Ypk1 by phosphorylating its C-terminal segment at multiple sites, two of which are highly conserved (the so-called "turn" and "hydrophobic" motifs), but all of which are required for maximal Ypk1 catalytic activity and for Ypk1 stability (Leskoske et al. 2017). Ypk1 in turn phosphorylates multiple substrates that modulate PM lipid and protein composition (for review, see Gaubitz et al. 2016; Roelants et al. 2017a). It also has been reported (Nomura and Inoue 2015) that TORC2 phosphorylates C-terminal turn-like and hydrophobiclike motifs in Pkc1, an AGC protein kinase that controls

(C) 2018 Leskoske et al. This article, published in Genes \& Development, is available under a Creative Commons License (Attribution 4.0 International), as described at http://creativecommons.org/licenses/by/4.0/. 
the cell wall integrity (CWI) pathway (Levin 2011); however, whether TORC2-mediated phosphorylation of Pkc1 affects its localization, stability, or activity has not been demonstrated.

Conditions that perturb the PM stimulate TORC2-mediated phosphorylation of Ypk1, including sphingolipid depletion (Roelants et al. 2011), hypotonic stress (Berchtold et al. 2012; Niles et al. 2012), heat shock (Sun et al. 2012), and elevated exogenous acetic acid (Guerreiro et al. 2016), whereas hyperosmotic stress inhibits TORC2mediated phosphorylation of Ypk1 (Lee et al. 2012; Muir et al. 2015). How TORC2 "senses" different stresses could, in some cases, involve direct feedback control. For example, sphingolipids seem to negatively regulate TORC2 because treatment of cells with a potent inhibitor (myriocin/ISP-1) of L-serine:palmitoyl-CoA C-palmitoyltransferase, the enzyme that catalyzes the first committed step in sphingolipid biosynthesis, causes up-regulation of TORC2-dependent and Ypk1-mediated phosphorylation of proteins whose modification markedly stimulates the rate of sphingolipid production (Roelants et al. 2011; Muir et al. 2014).

In addition, however, other pathways that sense PM or cell wall stress may exert some of their effects via regulation of TORC2 function. In this regard, two mitogenactivated protein kinase (MAPK) pathways in yeast sense and respond to stresses that challenge the cell envelope: the high-osmolarity glycerol (HOG) pathway, whose terminal MAPK is Hog1 (Saito and Posas 2012), and the CWI pathway, whose terminal MAPK is Slt2/Mpk1 (Levin 2011). Hog 1 activation, which can occur via a branch dependent on PM sensor $\operatorname{Sln} 1$ or a branch dependent on PM sensor Sho1, is required for cell survival under hypertonic conditions (Saito and Posas 2012) but can also be evoked by other environmental insults, including both heat shock (Winkler et al. 2002) and cold shock (Panadero et al. 2006), citric acid stress (Lawrence et al. 2004), hypoxia (Hickman et al. 2011), glucose starvation (Vallejo and Mayinger 2015), and, most notably from the perspective of this study, sphingolipid depletion (Tanigawa et al. 2012). Slt2 activation, which is initiated via stretch sensors anchored in both the PM and the cell wall (Wsc1, Mid2, and others) (Levin 2011), is required for cell survival under hypotonic conditions (Davenport et al. 1995) but can also be elicited by heat shock (Kamada et al. 1995) and agents that directly damage the cell wall, such as zymolyase, Calcofluor White, or Congo Red (de Nobel et al. 2000; García et al. 2004; Rodríguez-Peña et al. 2013). When activated, the stretch sensors recruit to the PM and activate Rom2 and/or Tus1-guanine nucleotide exchange factors (GEFs) for the Rhol GTPase. GTP-bound Rhol then binds to and activates Pkc1, which in turn activates the CWI MAPK cascade (Levin 2011). Rhol and its GEFs also localize at sites of polarized growth to promote cell wall glucan synthesis, actin cytoskeleton organization, and targeting of secretory vesicles to growth sites (Perez and Rincón 2010).

Significant cross-talk exists between the HOG and CWI pathways (Hahn and Thiele 2002; García-Rodríguez et al. 2005; Fuchs and Mylonakis 2009; García et al. 2009) and between these MAPK pathways and TORC2 function. For example, down-regulation of TORC2-Ypk1 signaling and activation of Hogl act in concert to maintain cell viability during hyperosmotic stress (Muir et al. 2015). In addition, one of the first functions reported for Tor2 (the TOR subunit unique to TORC2) was proper polarization of the actin cytoskeleton (Schmidt et al. 1996), linking Tor2 to Rhol and the CWI pathway. Indeed, various means to stimulate CWI pathway output rescue the inviability and actin polarization defects associated with loss of TORC2 or Ypk1 function (Helliwell et al. 1998; Roelants et al. 2002). In addition, TORC2-Ypk1 signaling, through its role in regulating lipid composition and organization, influences PM localization of Rhol and Rom2 (Niles and Powers 2014; Hatakeyama et al. 2017).

In this study, we sought to establish whether the CWI MAPK pathway directly modulates TORC2-Ypk1 signaling, given the cumulative evidence for the interconnections between them. As documented here, we found that activation of the CWI pathway MAPK results in direct Slt2-mediated phosphorylation of TORC2 subunits and that these modifications down-regulate TORC2Ypk1 signaling. By pinpointing the molecular basis of this negative regulation, our results provide new mechanistic insight into how TORC2 senses cell envelope stress.

\section{Results}

\section{TORC2 subunits are targets of the S1t2 MAPK}

TORC2 is a 1.4-MDa dimeric complex in which each protomer is a hetero-oligomer comprising Tor2, Lst8, Avo1, Avo2, Avo3, and either Bit2 or Bit61 (Wullschleger et al. 2005; Karuppasamy et al. 2017). Phosphoproteomic analyses (for compilations, see the Saccharomyces Genome Database, https://www.yeastgenome.org) have detected in vivo phosphorylation of -SP- and -TP- sites in four of the six TORC2 subunits (Fig. 1A). Most strikingly, nine such sites are present in Avo2, and four confirmed sites cluster at its C-terminal end; 11 sites are present in Avo3, and five confirmed sites cluster at its N-terminal end (Fig. 1A). Both Hog1 and Slt2 phosphorylate only -SP- or -TP- motifs (Mok et al. 2010), just like the proline-directed Ser- or Thr-specific MAPKs in other eukaryotes (Roux and Blenis 2004).

To determine whether any -SP- or -TP- sites in TORC2 subunits are direct targets of the Slt2 MAPK, we focused initially on Avo2. As expected for a phosphoprotein, Avo2 exists, even under basal conditions, in a series of isoforms of distinct mobility, as resolved by Phos-tag SDS-PAGE (Fig. 1B), a technique in which the degree of retardation of a protein reflects the extent of its phosphorylation (Kinoshita et al. 2009). A convenient method to activate Slt2 in the absence of an external stimulus is overexpression of a constitutively active $\mathrm{Pkcl}$ allele, Pkc1(R398A R405A K406A) (referred to here as PKC1*) (Martín et al. 2000), which is hyperactive because residues critical for the inhibitory function of its pseudosubstrate sequence have been mutated. After induction of $P K C 1^{*}$ 
expression, Avo2 became hyperphosphorylated, and the increase in Avo2 phosphorylation coincided with the kinetics of appearance of active Slt2 (Fig. 1B). Overexpression of a catalytically inactive ("kinase-dead" [KD]) derivative, $\mathrm{Pkc1}^{*}(\mathrm{D} 949 \mathrm{~A})$, to the same level as Pkc1* (Fig. 1C) did not activate Slt2 or alter Avo2 phosphorylation (Fig. 1B).

In addition to robust $S 1 \mathrm{t} 2$ activation, we noted that overexpression of $\mathrm{Pkcl}^{*}$ also evoked $\mathrm{Hog} 1$ activation, in agreement with other observations demonstrating cross-talk between these two MAPK pathways (Hahn and Thiele 2002; García-Rodríguez et al. 2005; Fuchs and Mylonakis 2009; García et al. 2009). Therefore, to distinguish which MAPK was primarily responsible for the Avo2 hyperphosphorylation induced by $\mathrm{Pkcl}^{*}$ overexpression, we repeated these experiments in both slt2 $\Delta$ and hog1 $1 \Delta$ mutants. We found that the $\mathrm{Pkcl}^{*}$-induced mobility shift was markedly reduced in slt $2 \Delta$ cells but not in hog $1 \Delta$ cells (Fig. 1D). Moreover, to confirm that the Pkc1*-induced

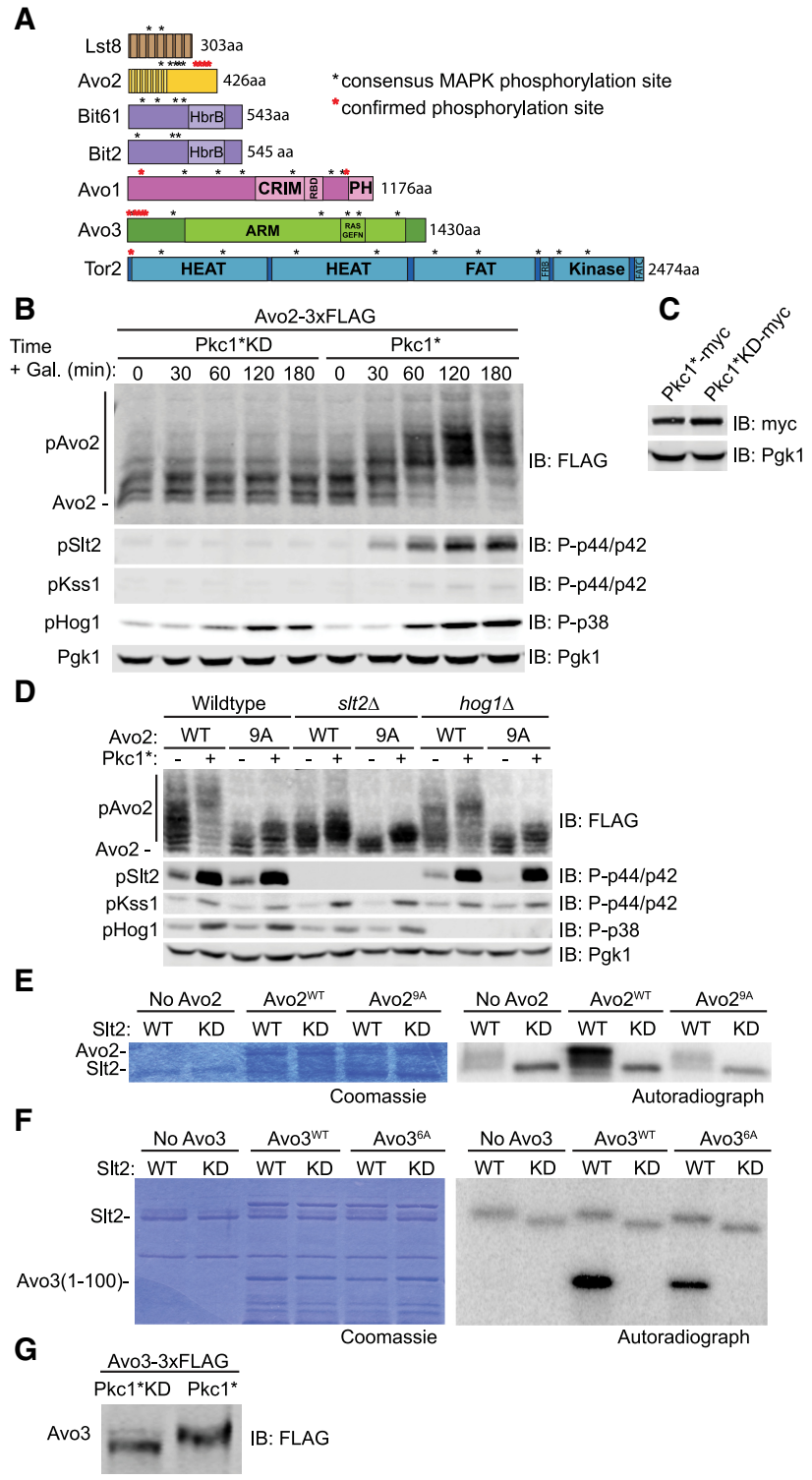

Slt2-mediated modifications were occurring on the -SP- and -TP- sites, the same experiments were repeated with an Avo2 allele (Avo2 $\left.{ }^{9 \mathrm{~A}}\right)$ in which all nine sites were mutated to Ala. Indeed, absence of these sites prevented $\mathrm{Pkcl}^{*}$-induced hyperphosphorylation of Avo2 (Fig. 1D). Although Slt2 appears to be the major MAPK responsible for $\mathrm{Pkcl}^{*}$-induced hyperphosphorylation of Avo2, these modifications were not completely abrogated in slt2 $\Delta$ cells and were modestly reduced in hog $1 \Delta$ cells compared with wild-type cells, suggesting that Hog1 makes a minor contribution to $\mathrm{Pkcl}^{*}$-induced hyperphosphorylation of Avo2.

In further support of the capacity of Slt2 to modify Avo2, wild-type Slt2 immuno-enriched from cells overexpressing $\mathrm{Pkc1}^{*}$-but not a catalytically deficient derivative, Slt2(K54R), purified in the same manner-robustly phosphorylated recombinant wild-type Avo2 in vitro,

Figure 1. MAPK Slt2 phosphorylates TORC2 subunits Avo2 and Avo3. (A) The primary structure of each indicated TORC2 subunit is depicted schematically, with domains labeled as in Gaubitz et al. (2016). (Black asterisk) An -SP- or -TP- site; (red asterisk) an -SP- and -TP- detectably phosphorylated in vivo in various phosphoproteomic analyses, as cataloged in the Saccharomyces Genome Database (http://www.yeastgenome.org). (B) Wild-type cells (JTY5336) carrying both a CEN plasmid expressing Avo2-3xFlag (pKL1) from the AVO2 promoter and a multicopy (2 um DNA) vector expressing either Pkc1* (pJT5660) or catalytically inactive $\mathrm{Pkc1}{ }^{*} \mathrm{KD}$ (pJEN12) from the GAL1 promoter were cultured to mid-exponential phase in selective minimal medium containing $2 \%$ raffinose and $0.2 \%$ sucrose. Expression of $\mathrm{Pkc}^{*}$ or $\mathrm{Pkc}^{*} \mathrm{KD}$ was induced by addition of galactose $(2 \% \mathrm{fi}-$ nal concentration). Cell samples were removed at the indicated times and lysed, the proteins in the resulting extracts were resolved by Phos-tag SDS-PAGE, and the indicated proteins were analyzed by immunoblotting with the appropriate antibodies, all as described in the Materials and Methods. Pgk1 was the loading control. $(C)$ As in $B$ except the cells carried plasmids expressing either Pkc1*-myc (pAEA376) or Pkc1*KD-myc (pJEN13), and the extracts were resolved by standard SDS-PAGE prior to immunoblotting. $(D)$ Wild-type cells (JTY5336) or otherwise isogenic

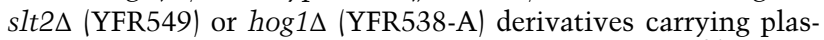
mids expressing either Avo2-3xFlag (pKL1) or Avo2 ${ }^{9 \mathrm{~A}}-3 \mathrm{xFlag}$ (pKL2), as indicated, as well as either empty vector (yEPlac112) or the same vector expressing $\mathrm{Pkc1}^{*}$ from the GAL1 promoter (pJT5660), as shown, were grown to mid-exponential phase in selective minimal medium containing $2 \%$ raffinose and $0.2 \%$ sucrose. After addition of galactose (2\% final concentration), the cells were cultured for $3 \mathrm{~h}$, harvested, and lysed, and the indicated proteins were analyzed as in $B$. (E) Activated wild-type Slt2 (pKL63) and kinase-dead (KD) Slt2 (pKL64) were purified from Saccharomyces cerevisiae as described in the Materials and Methods and incubated with $\left[\gamma^{-32} \mathrm{P}\right] \mathrm{ATP}$ and either GST-Avo ${ }^{\mathrm{WT}}$ (pKL16) or GST-Avo2 ${ }^{9 \mathrm{~A}}$ (pKL17) purified from Escherichia coli as described in the Materials and Methods. Reaction products were resolved by SDS-PAGE and analyzed by Coomassie blue staining (left) and autoradiography (right). (F) As in E, except the substrates were purified recombinant GST-Avo3(1-100) (pKL81) or GSTAvo3(1-100) ${ }^{6 \mathrm{~A}}$ (pKL82). (G) A strain (YFR617) expressing Avo3$3 x F l a g$ and carrying a plasmid expressing either $\mathrm{Pkc}^{*} \mathrm{KD}$ (pJEN12) or Pkc1* (pJT5660) from the GAL1 promoter was grown, induced with galactose for $2.5 \mathrm{~h}$, lysed, and analyzed by Phos-tag SDS-PAGE as in $B$. 
and this incorporation was eliminated in an Avo2 ${ }^{9 \mathrm{~A}} \mathrm{mu}-$ tant (Fig. 1E).

Likewise, Avo3(1-100), an N-terminal fragment containing six of the 11 -SP- and -TP- sites in Avo3 (five of which have been detected as phosphorylated in vivo), was robustly phosphorylated in vitro by wild-type Slt2 but not by the catalytically deficient Slt2 mutant, and incorporation was markedly reduced by mutation of the six sites to Ala (Fig. 1F). Moreover, as judged by its mobility shift, intact Avo3-3xFlag was phosphorylated in vivo upon overexpression of $\mathrm{Pkcl}^{*}$ but not upon overexpression of $\mathrm{Pkcl}^{*} \mathrm{KD}$ (Fig. 1G). Collectively, these results show that at least two core subunits of TORC2 are physiologically relevant substrates of the Slt2 MAPK.

\section{MAPK phosphorylation of Avo2 impairs TORC2 activity}

Unlike Avo3 (1430 residues), which is essential for TORC2 function because it has an important structural role (Wullschleger et al. 2005; Karuppasamy et al. 2017), Avo2 is a relatively small (426 residues) peripherally located nonessential subunit of TORC2, with the highest overall density of -SP- and -TP- sites (Fig. 1A), suggesting that its Slt2-mediated phosphorylation might have a readily detectable regulatory role. To examine this possibility, in addition to wild-type Avo2 and the MAPK site-deficient Avo $2^{9 \mathrm{~A}}$ mutant, we also generated a phosphomimetic allele, Avo $2^{9 \mathrm{E}}$. First, we found that all three proteins were expressed at comparable steady-state levels (Fig. 2A); thus, the absence or presence of MAPK phosphorylation does not affect Avo2 stability. Strikingly, however, even though Avo2 is nonessential for cell viability under nonstress conditions, we found that Avo2 is essential for cell survival during myriocin-induced sphingolipid depletion (Fig. 2B, top row), a condition in which cells require full TORC2-mediated activation of Ypk1 to survive (Roelants et al. 2011; Leskoske et al. 2017). Furthermore, compared with cells expressing wild-type Avo2 or Avo $2^{9 \mathrm{~A}}$, cells expressing Avo ${ }^{9 \mathrm{E}}$ were much more sensitive to the growth inhibitory effect of myriocin (Fig. 2B), suggesting that, like avo2s cells, they are deficient in TORC2-dependent Ypk1 activation. In direct support of that conclusion, the myriocin sensitivity of both the avo2 $\Delta$ mutant and Avo $2^{9 \mathrm{E}}$-expressing cells was rescued by coexpression of a Ypk1 allele, Ypk1(D242A), that is fully active in the absence of TORC2 phosphorylation (Fig. 2C; Kamada et al. 2005; Roelants et al. 2011; Leskoske et al. 2017). These findings indicate that Avo2 is required for optimal TORC2 function and that phosphorylation interferes with Avo2 function, resulting in down-regulation of TORC2 activity.

These conclusions were confirmed by monitoring TORC2 activity in vivo. We documented previously that TORC2 phosphorylates Ypk1 at multiple sites that lie within 40 residues of its $\mathrm{C}$ terminus and that full phosphorylation of these sites is necessary for both maximal Ypk1 activity and Ypk1 stability (Leskoske et al. 2017). Therefore, to assess TORC2 activity in vivo, we examined phosphorylation of Ypk1 at four of its C-terminal TORC2dependent phosphorylation sites using Phos-tag SDS-
A

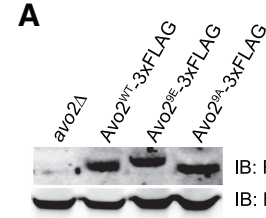

B

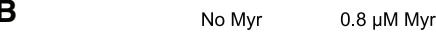

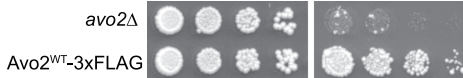
Avo2 ${ }^{9 E}-3 \times F L A G O O F$ B: Pgk1

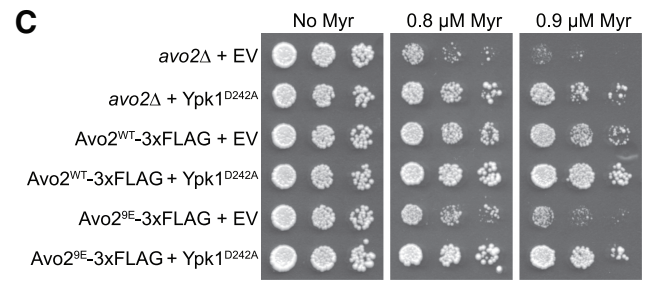

D
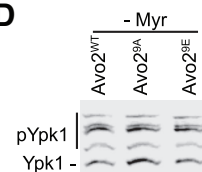

Ypk

$\mathbf{F}$

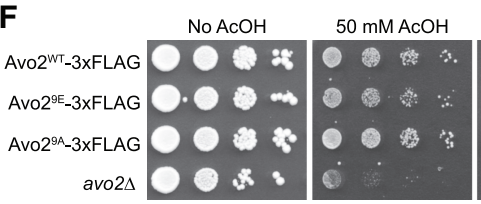

E
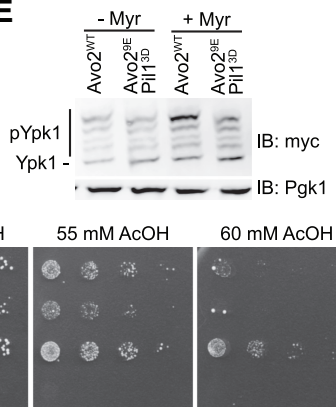

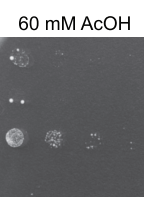

Figure 2. Phosphorylation of Avo2 at its MAPK sites impairs TORC2 activity. (A) Otherwise isogenic strains JTY7318 (avo2s), yKL32 (Avo2 $\left.{ }^{\mathrm{WT}}-3 \times \mathrm{xFlag}\right)$, yKL34 (Avo2 ${ }^{\mathrm{EE}}-3 \mathrm{xFlag}$ ), and yKL33 (Avo2 ${ }^{9 \mathrm{~A}}-3 \mathrm{xFlag}$ ) were grown to mid-exponential phase in rich medium, harvested, and lysed, and the resulting extracts were resolved by SDS-PAGE and analyzed by immunoblotting, as described in the Materials and Methods. Pgk1 was the loading control. (B) Overnight cultures of the strains in $A$ were adjusted to $A_{600 \mathrm{~nm}}=0.1$, spotted undiluted and in a series of fivefold serial dilutions on YPD plates either lacking or containing $0.8 \mu \mathrm{M}$ myriocin, incubated for $3 \mathrm{~d}$ at $30^{\circ} \mathrm{C}$, and then imaged. $(C)$ Strains JTY7318 (avo2s), yKL32 (Avo2 $\left.{ }^{\mathrm{WT}}-3 \times \mathrm{xFlag}\right)$, or yKL34 (Avo2 $2^{\text {'E }}$ $3 x F l a g$ ) expressing either empty vector (pRS315) or Ypk1 ${ }^{\mathrm{D} 242 \mathrm{~A}}$ myc from the same vector (pFR234) were adjusted to $A_{600 \mathrm{~nm}}=$ 0.1 , spotted as in $B$ but on SCD-L-T plates lacking or containing myriocin at the indicated concentrations, and imaged after incubation for $3 \mathrm{~d}$ at $30^{\circ} \mathrm{C}$. (D) Wild-type (BY4742), Avo2 ${ }^{9 \mathrm{~A}}$ (YFR528), or Avo $2^{9 \mathrm{E}}$ (yKL31) strains expressing Ypk $1^{5 \mathrm{~A}}$-myc from its native promoter on a CEN plasmid (pFR246) were cultured to mid-exponential phase in selective minimal medium and then treated with vehicle (methanol) or $1.25 \mu \mathrm{M}$ myriocin for $2 \mathrm{~h}$. After harvesting, whole-cell lysates were prepared, resolved by SDS-PAGE, and analyzed as described in the Materials and Methods. A representative of three independent experiments is shown. (E) As in $D$, except strains BY4742 and Avo2 ${ }^{9 \mathrm{E}}$ Pill $^{3 \mathrm{D}}$ (YFR641) were used. $(F)$ Overnight cultures of the strains in $A$ were adjusted to $A_{600 \mathrm{~nm}}=0.1$, spotted undiluted and in a series of 10 -fold serial dilutions on SCD plates either lacking or containing acetic acid at the indicated concentrations, and imaged after incubation for 3 d at $30^{\circ} \mathrm{C}$.

PAGE. Treatment with myriocin, a condition that activates TORC2 activity in wild-type cells, failed to stimulate TORC2 phosphorylation of Ypk1 in Avo2 ${ }^{9 \mathrm{E}}$ expressing cells (Fig. 2D). As presented further below, Avo2 has also been found in association with PM 
structures called eisosomes (Douglas and Konopka 2014), and Pill, a major eisosome component, has been shown to be another target of Slt2 (Mascaraque et al. 2013). Hence, we also examined Ypk1 phosphorylation in cells expressing both $\mathrm{Avo} 2^{9 \mathrm{E}}$ and Pill ${ }^{3 \mathrm{D}}$ and again found that, unlike in wild type or cells expressing Pil1 ${ }^{3 \mathrm{D}}$ alone (Supplemental Fig. S1), myriocin treatment failed to stimulate TORC2 phosphorylation of Ypk1 in the cells expressing both Avo $2^{9 \mathrm{E}}$ and Pil1 ${ }^{3 \mathrm{D}}$ (Fig. 2E). These additional observations verify that it is phosphorylation of Avo2 that makes a major contribution to interfering with TORC2 function.

Another stress that cells require TORC2-mediated Ypk1 activation to survive is exposure to elevated exogenous acetic acid (Guerreiro et al. 2016). As with myriocin treatment, avo2 $\Delta$ cells were markedly more sensitive to this stress than wild-type cells, and cells expressing Avo $2{ }^{9 \mathrm{E}}$ were detectably more sensitive than cells expressing either wild-type Avo2 or especially Avo2 ${ }^{9 \mathrm{~A}}$ (Fig. 2F). These results again show that Avo2 is required for full TORC2 activity and that MAPK phosphorylation of Avo2 impairs TORC2 function.

\section{Slt2 MAPK action down-regulates TORC2 function}

As assessed by Phos-tag SDS-PAGE, after induction of $\mathrm{Pkc}^{*} \mathrm{KD}$, there was no change in the pattern of TORC2dependent Ypk1 isoforms over the course of $2.5 \mathrm{~h}$, whereas after induction of $\mathrm{Pkcl}^{*}$, there was a decrease in the slowest mobility (most highly phosphorylated) isoforms, concomitant with the appearance of activated Slt2 (Fig. 3A), as well as a marked reduction of total Ypk1 protein (Fig. 3A,B), indicating that activation of the CWI pathway prevented TORC2-mediated phosphorylation of Ypk1. The observed $\mathrm{Pkcl}^{*}$-induced down-regulation of TORC2-mediated Ypk1 phosphorylation and loss of Ypk1 protein was largely prevented in a slt2 $\Delta$ mutant but not in $h o g 1 \Delta$ or kss1 $1 \Delta$ single mutants or a hog $1 \Delta$ kss1 $\Delta$ double mutant (Fig. 3C). We examined a kss1 $\Delta$ mutation alone or in combination with other MAPK-null mutations because we noted that overexpression of $\mathrm{Pkc1}^{*}$ sometimes mildly increased the amount of dually phosphorylated (activated) Kss1 MAPK (Fig. 1D). However, compared with the slt2 $\Delta$ cells, the levels of Ypk1
A

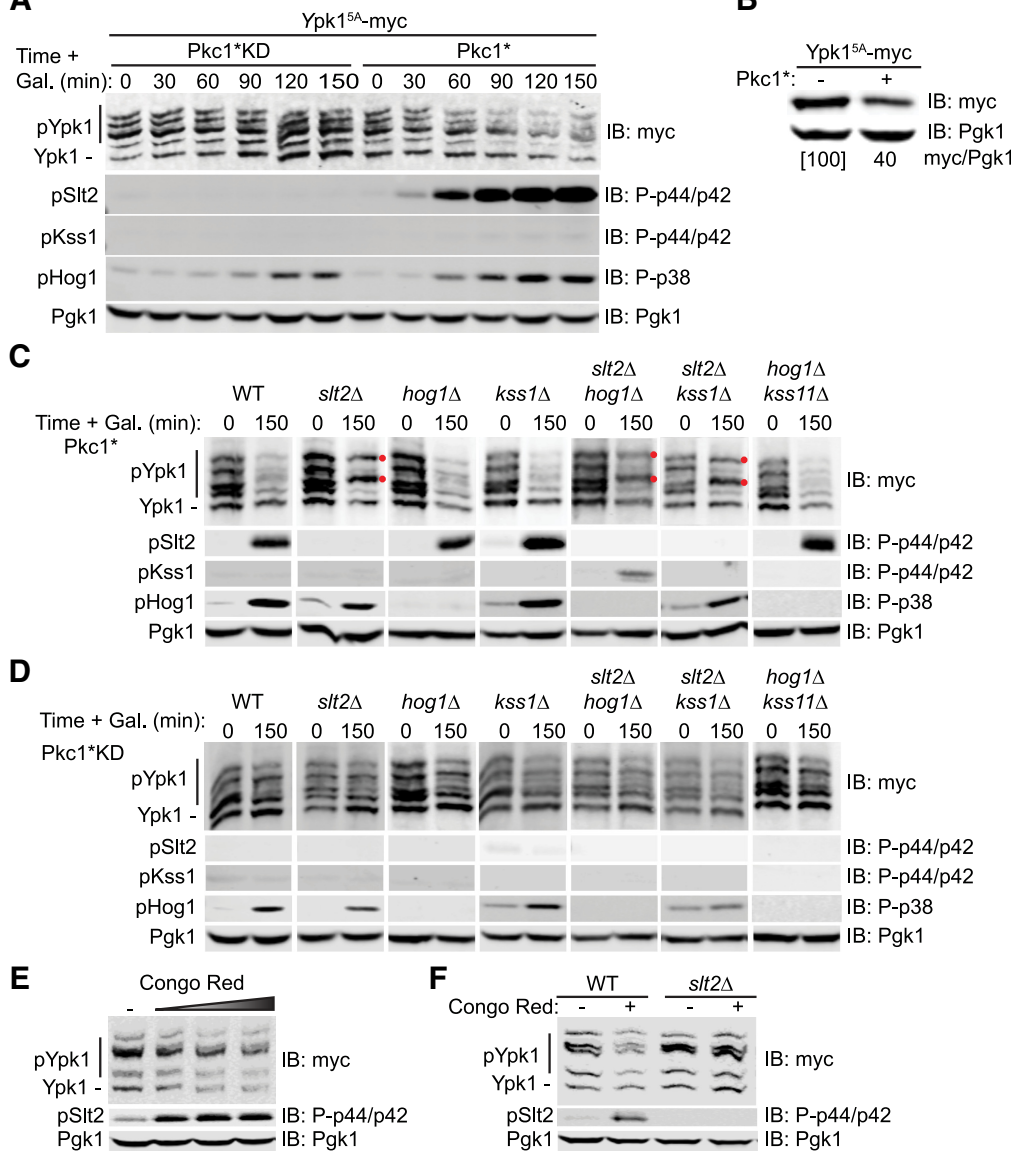

Figure 3. Activation of MAPK Slt2 down-regulates TORC2-mediated Ypk1 phosphorylation. (A) Strain JTY5336 expressing Ypk1 ${ }^{5 \mathrm{~A}}$-myc from its native promoter on a CEN plasmid (pFR246) and expressing from the GAL1 promoter on plasmids either $\mathrm{Pkc1}^{*} \mathrm{KD}$ (pJEN12) or Pkc1* (pJT5660), as indicated, were cultured to mid-exponential phase in selective minimal medium containing $2 \%$ raffinose and $0.2 \%$ sucrose and induced by addition of galactose $(2 \%$ final concentration), and samples taken at the indicated time points were lysed and analyzed by Phos-tag SDS-PAGE and immunoblotting, as described in the Materials and Methods. Pgk1 was the loading control. (B) Strain JTY5336 expressing Ypk1 ${ }^{5 \mathrm{~A}}$-myc from its native promoter on a CEN plasmid (pFR246) and carrying either empty vector $(-)$ (yEPlac112) or expressing $\mathrm{Pkcl}^{*}(+)$ from the GAL1 promoter on the same vector were grown as in $A$ and harvested $2 \mathrm{~h}$ after galactose induction, and the relative level of Ypk1 protein was determined and normalized to the Pgk1 loading control (ratio was set at 100 for the empty vector control) by SDS-PAGE and immunoblotting. A representative image is shown for an experiment that was repeated three independent times. $(C)$ Strains JTY5336 $\left(\mathrm{HOG}^{+} \mathrm{KSS1}^{+} \mathrm{SLT2} \mathrm{2}^{+}\right), \quad$ YFR549

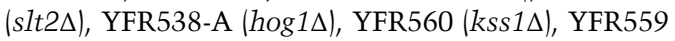

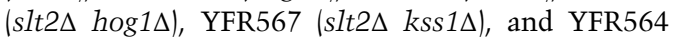
(hog $1 \Delta$ kss $1 \Delta$ ) expressing Ypk1 ${ }^{5 \mathrm{~A}}$-myc from its native promoter on a CEN plasmid (pFR246) and coexpressing $\mathrm{Pkcl}^{*}$ from the GAL1 promoter on a plasmid (pJT5660) were cultured to mid-exponential phase in selective minimal medium containing $2 \%$ raffinose and $0.2 \%$ sucrose and induced by addition of galactose ( $2 \%$ final concentration), and, after $2.5 \mathrm{~h}$, samples were lysed and analyzed as in $A$. Pgk 1 was the loading control. (Red dots) TORC2-dependent isoforms of Ypk1 that persist. $(D)$ As in $C$, except the cells coexpressed Pkc1*KD from the GAL1 promoter on the plasmid (pJEN12). (E) Wild-type (BY4741) cells expressing Ypk1 ${ }^{5 \mathrm{~A}}$-myc from its native promoter on a CEN plasmid (pFR246) were cultured to mid-exponential phase in selective minimal medium and then treated with increasing amounts of Congo $\operatorname{Red}(0,10,20$, or $40 \mu \mathrm{g} / \mathrm{mL}$ final concentration) for $2 \mathrm{~h}$. After harvesting, whole-cell lysates were prepared, resolved by SDS-PAGE, and analyzed as described in the Materials and Methods. (F) Same as in E, except that wild-type (JTY5336) and slt2 (YFR549) strains were used, and, when added, Congo Red was added at $20 \mu \mathrm{g} / \mathrm{mL}$ final concentration. 
phosphorylation or Ypk1 protein were not further enhanced in hog $1 \Delta$ slt2 $2 \Delta$ or kss $1 \Delta$ slt $2 \Delta$ double mutants (Fig. 3 C). Most revealingly, in every case where Slt2 is absent, two of the most prominent and highly TORC2 phosphorylated Ypk1 isoforms persisted (Fig. 3C, red dots). Thus, Slt2 is the MAPK primarily responsible for inhibiting TORC2-mediated Ypk1 phosphorylation.

It has been reported that $\mathrm{Pkc} 1$ is a substrate of TORC2 (Nomura and Inoue 2015); hence, it was possible that overexpression of even $\mathrm{Pkcl}^{*} \mathrm{KD}$ might titrate away a sufficient amount of TORC2 to interfere with its efficient phosphorylation of Ypk1. However, this was not the case because, in either wild-type cells or all of these same mutants, induction of $\mathrm{Pkcl}^{*} \mathrm{KD}$ had little or no effect on the pattern of the Ypk1 isoforms (Fig. 3D). Furthermore, treatment of cells expressing normal Pkc1 and Slt2 at their endogenous levels with the cell wall-perturbing dye Congo Red, a physiological condition that has been demonstrated to elicit Pkc1-dependent Slt2 activation (de Nobel et al. 2000), resulted in down-regulation of TORC2 function (Fig. 3E), and the down-regulation was dependent on Slt2 (Fig. 3F).

\section{TORC2 is down-regulated during sustained $\operatorname{Sin} 1$ inactivation}

$\operatorname{Sln} 1$ is a histidine kinase similar to those in bacterial two-component signaling systems, and its inactivation, as occurs during hyperosmotic shock, leads to activation of the Hog1 MAPK (Saito and Posas 2012). In a global screen of the Saccharomyces cerevisiae kinome, we found that, like a tor $^{\text {ts }}$ mutant (Leskoske et al. 2017), when a $\sin 1^{t s}$ mutant was shifted to the restrictive temperature, there was a dramatic loss of TORC2-dependent Ypk1 phosphorylation (Supplemental Fig. S2A) that was accompanied by hyperphosphorylation of Avo2 (Supplemental Fig. S2B), indicating that, like activation of the CWI MAPK pathway, activation of the HOG MAPK pathway down-regulates TORC2 function and does so via a similar mechanism.

To confirm this conclusion and inactivate $S \ln 1$ independently of any external temperature or hypertonic stress, we constructed a strain expressing the plant F-box protein Tirl and an allele of Sln 1 tagged with a modified auxin-inducible degron (AID*), which should cause Sln1 to be degraded in an SCF-mediated manner upon auxin addition (Morawska and Ulrich 2013). Adding a synthetic auxin (1-naphthaleneacetic acid [1-NAA]) to cells expressing untagged $S \ln 1$ had no effect on the level of Sln1 or on the Ypk1 isoforms present and did not activate either Hog1 or Slt2 (Fig. 4A, left). The same treatment of cells expressing AID*-tagged Sln 1 resulted in its rapid degradation, as expected, as well as the loss of the Ypk1 isoforms, concomitant with the activation of both Hog1 and Slt2 (Fig. 4A, right), in agreement with prior work showing that Hog1 activation leads to Slt2 activation (García-Rodríguez et al. 2005). Thus, we again found that MAPK activation coincided with down-regulation of TORC2 activity, as judged by the dramatic drop in Ypk1 C-terminal phosphorylation and protein level.

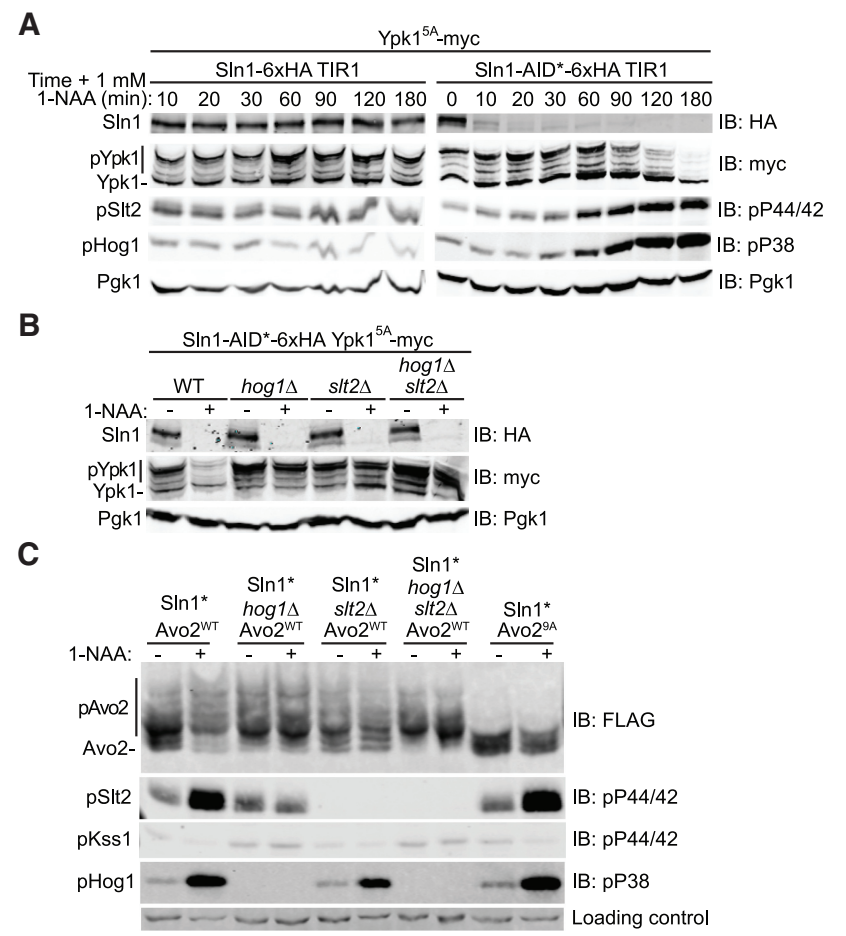

Figure 4. TORC2 function is down-regulated after $S \ln 1$ degradation. (A) Strains yKL15 (Sln1-6xHA TIR1) or yKL18 (Sln1-AID*6xHA TIR1) expressing Ypk1 ${ }^{5 \mathrm{~A}}$-myc from its native promoter on a $C E N$ plasmid (pFR246) were grown to mid-exponential phase in phosphate-buffered selective minimal medium $(\mathrm{pH}$ 6.2) and treated with $1 \mathrm{mM}$ 1-NAA (final concentration), and samples were withdrawn at the indicated times, lysed, subjected to Phos-tag SDS-PAGE to resolve Ypk1 phosphorylation and to standard SDS-PAGE to resolve the other proteins, and analyzed by immunoblotting as described in the Materials and Methods. (B) Strains yKL18 (Sln1-AID*-6xHA TIR1 HOG $\left.1^{+} S L T 2^{+}\right)$, yKL20

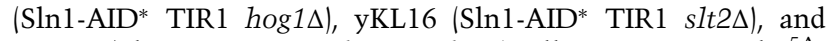

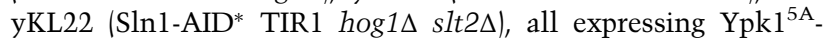
myc from its native promoter on a $C E N$ plasmid (pFR246), were grown as in $A$, treated with either solvent alone (DMSO) or 1 mM 1-NAA (final concentration) in the same volume of solvent for $90 \mathrm{~min}$, harvested, lysed, and analyzed as in $A$. $(C)$ The same strains as in $B$, except expressing either Avo $2^{\mathrm{WT}}-3 x$ Flag (pKL1) or Avo2 $2^{9 \mathrm{~A}}-3 \mathrm{xFlag}$ (pKL2), as indicated, were grown and treated as in $B$, except that the incubation with 1-NAA was for $2 \mathrm{~h}$, and samples of each culture were analyzed as in $A$, with Avo2 phosphorylation resolved by Phos-tag SDS-PAGE, and the other proteins resolved by standard SDS-PAGE.

Consistent with the cross-talk by which activation of Hog1 leads to activation of Slt2 (García-Rodríguez et al. 2005), the drastic reduction in Ypk1 C-terminal phosphorylation and protein level caused by $\operatorname{Sin} 1$ degradation was ameliorated in either a hog $1 \Delta$ mutant or a slt $2 \Delta$ mutant and was not further enhanced in a hog $1 \Delta$ slt2 $\Delta$ double mutant (Fig. 4B). Moreover, similar to what we observed upon $\mathrm{Pkc}^{*}$-induced CWI pathway activation, activation of the HOG pathway by Sln 1 degradation markedly stimulated phosphorylation of Avo2, as judged by the shift to a spectrum of slower mobility isoforms in the Phos-tag gel (Fig. 4C, left two lanes), and no such shift was observed 
for the Avo $2^{9 \mathrm{~A}}$ mutant, indicating that these modifications were occurring on its -SP- and -TP- sites (Fig. 4C, right two lanes). Furthermore, there was no change in the pattern of Avo2 isoforms upon Sln1 degradation in cells lacking either Hog 1 or Slt2 (or both) (Fig. 4C, middle lanes), indicating that Avo2 phosphorylation was MAPKdependent. Importantly, activation of Hog1 alone (upon degradation of $S \ln 1$ in slt2 $\Delta$ cells) was not sufficient to down-regulate TORC2-mediated Ypk1 phosphorylation (Fig. 4B) or stimulate Avo2 phosphorylation (Fig. 4C), indicating that Slt2 is the MAPK responsible for these effects and that, in this case, the primary role of activated Hog 1 is to stimulate production of activated Slt2.

Interestingly, we documented previously that when the HOG pathway is activated by exposure of cells to $1 \mathrm{M}$ sorbitol, a cell-impermeable osmolyte that robustly but transiently activates Hog1 (Saito and Posas 2012), there is rapid and transient collapse of the TORC2-dependent Ypk1 isoforms, but that response still occurs even in hog1s cells (Lee et al. 2012; Muir et al. 2015). Although treatment with $1 \mathrm{M}$ sorbitol activates Hog1, it does not activate Slt2, and the collapse of the TORC2-dependent Ypk1 isoforms still occurs in cells lacking either Slt2 or Hog1 (or both) (Supplemental Fig. S3). Thus, in contrast to the Slt2-dependent down-regulation of TORC2-mediated Ypk1 phosphorylation that occurs upon prolonged inactivation of Sln1, the loss of the TORC2-dependent Ypk1 isoforms caused by exposure to $1 \mathrm{M}$ sorbitol occurs in a MAPK-independent manner. Possibly, the hypertonic shock caused by $1 \mathrm{M}$ sorbitol activates an as yet to be identified phosphatase. In any event, both acute and chronic stimulation of the HOG pathway leads to downregulation of TORC2-Ypk1 signaling, albeit by different mechanisms.

\section{MAPK phosphorylation of Avo2 alters its subcellular localization}

Studies of various integral and peripheral PM proteins in yeast have shown that different components often reside in discrete microdomains (Spira et al. 2012). Many permeases, such as the arginine transporter Can1, colocalize in a membrane compartment called the "MCC" that is congruent with eisosomes (Strádalová et al. 2009), protein-coated PM invaginations whose major constituents include Pill, an F-BAR (FCH/bin-amphiphysin-rvs) domain-containing protein (Karotki et al. 2011). Initially, it was reported that all of the components of TORC2, including Avo2, reside in a PM microdomain dubbed the "MCT" that is distinct from eisosomes (Berchtold and Walther 2009). However, subsequent work demonstrated that Avo2 partitions between TORC2 and eisosomes (Bartlett et al. 2015). For these reasons, and having established that MAPK phosphorylation does not affect Avo2 stability (Figs. 2A, 5C), to explain how MAPK phosphorylation of Avo2 contributes to down-modulation of TORC2 activity, we examined whether MAPK phosphorylation of Avo2 affected its subcellular localization.

Toward that end, we expressed either Avo2, Avo2 ${ }^{9 \mathrm{~A}}$, or Avo $2^{9 \mathrm{E}}$ (each tagged at its $\mathrm{C}$ terminus with $\mathrm{mNeon}$ Green
[mNG]) (Shaner et al. 2013) from its endogenous locus on chromosome XIII in cells coexpressing the eisosome marker Pill (tagged at its C terminus with red fluorescent protein [RFP]) (Fradkov et al. 2000) from its endogenous locus on chromosome VII. We document (1) that Avo2mNG is fully functional (Supplemental Fig. S4) and (2) that both Avo2-mNG and Pill-RFP displayed a punctate distribution at the PM and that these puncta were often, but not always, congruent (Fig. 5A, left), as we resolved and quantified using CellProfiler and appropriate masking (Supplemental Fig. S5). The same was observed for Avo ${ }^{9 \mathrm{~A}}$-mNG and Pill-RFP (Fig. 5A, middle). However, there were significant differences found in the cells expressing Avo $2^{9 \mathrm{E}}-\mathrm{mNG}$ and Pill-RFP. Generally, the Avo ${ }^{9 \mathrm{E}}$-containing puncta were smaller and less prominent (Fig. 5A, right), and their overall mean pixel intensity was significantly lower than in cells expressing Avo2 or Avo $2^{9 \mathrm{~A}}$ (Fig. 5B) despite the fact that the total amount of Avo2, Avo $2^{9 \mathrm{~A}}$, and $\mathrm{Avo} 2^{9 \mathrm{E}}$ in each of these cells was very similar (Fig. 5C), and the intensity of the Pill-RFP foci remained essentially unchanged (Fig. 5A,B). Moreover, the decrease in the intensity of the Avo $2^{9 \mathrm{E}}$-containing PM puncta was the same regardless of whether the Avo2 ${ }^{9 \mathrm{E}}$ colocalized with Pill (Fig. 5D) or was located at distinct PM sites, presumably with TORC2 (Fig. 5E). Taken together, these findings suggest that MAPK phosphorylation promotes dissociation of Avo2 from either of its two PM sites: eisosomes and TORC2.

To confirm that conclusion for TORC2 specifically by an independent biochemical approach, we immuno-isolated TORC2 (in which the Avo3 subunit carried a 3xFlag epitope tag) from the cells expressing Avo2, Avo2 ${ }^{9 \mathrm{~A}}$, or Avo $2^{9 \mathrm{E}}$ and analyzed the amount of Avo 2 present in these complexes. In the immunoprecipitates obtained using anti-Flag antibodies, equivalent amounts of both Avo3$3 x$ Flag and the Tor2 catalytic subunit were found regardless of whether the cells expressed Avo2, Avo2 ${ }^{9 A}$, or Avo2 ${ }^{9 \mathrm{E}}$ (Fig. 5F). Likewise, in cells expressing Tor2$\mathrm{mNG}$ and Avo2, Avo2 ${ }^{9 \mathrm{~A}}$, or Avo2 ${ }^{9 \mathrm{E}}$, there was little or no effect on the amount of Tor2 present in PM-associated TORC2 (Supplemental Fig. S6). However, quantification of the immunoprecipitates showed that, compared with the amount of Avo2 or Avo2 ${ }^{\mathrm{A}}$ bound to TORC2, the amount of $\mathrm{Avo}^{\mathrm{PE}}$ was always reduced by $30 \%-40 \%$. Thus, in agreement with the observations made by fluorescence imaging, these results suggest that MAPK phosphorylation of Avo2 promotes its dissociation from TORC2.

\section{Discussion}

It has been unclear whether TORC2 adjusts its activity by serving itself as a direct sensor of perturbations to the cell envelope or responding to inputs from other stress-sensing pathways (or both). We demonstrated here, for the first time, that Slt2/Mpk1 (mammalian ortholog ERK5/ MAPK7) (Truman et al. 2006), the MAPK of the CWI pathway, phosphorylates two subunits of TORC2 (Avo2 and Avo3) and, as a result, down-regulates TORC2 
A

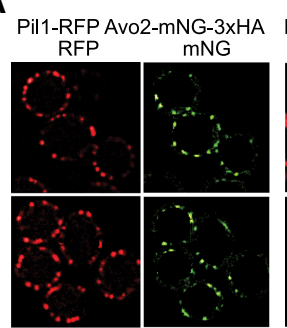

C

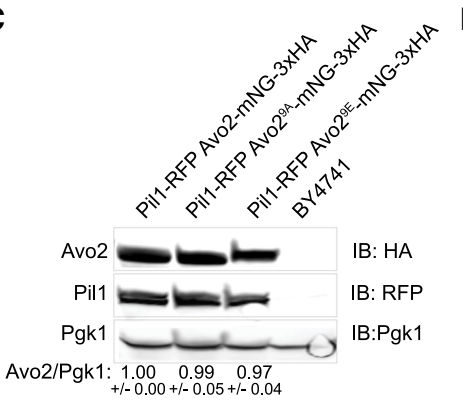

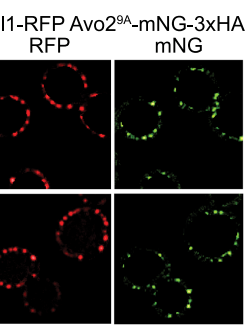

D

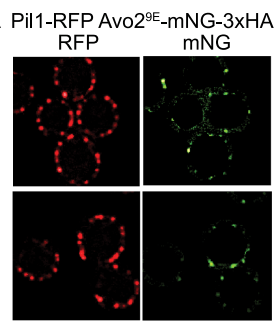

Avo2 colocalized with Pil1
B

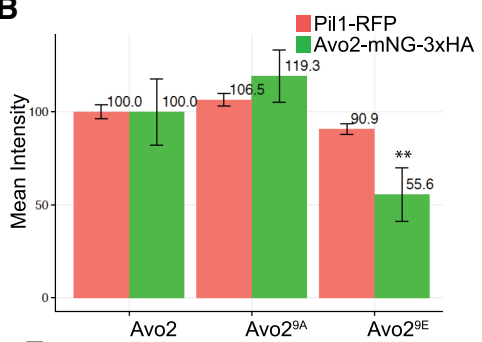

E

Avo2 not colocalized with Pil1
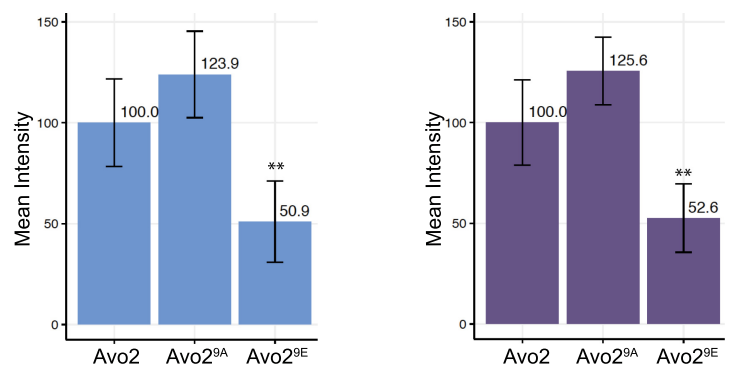

$\mathbf{F}$
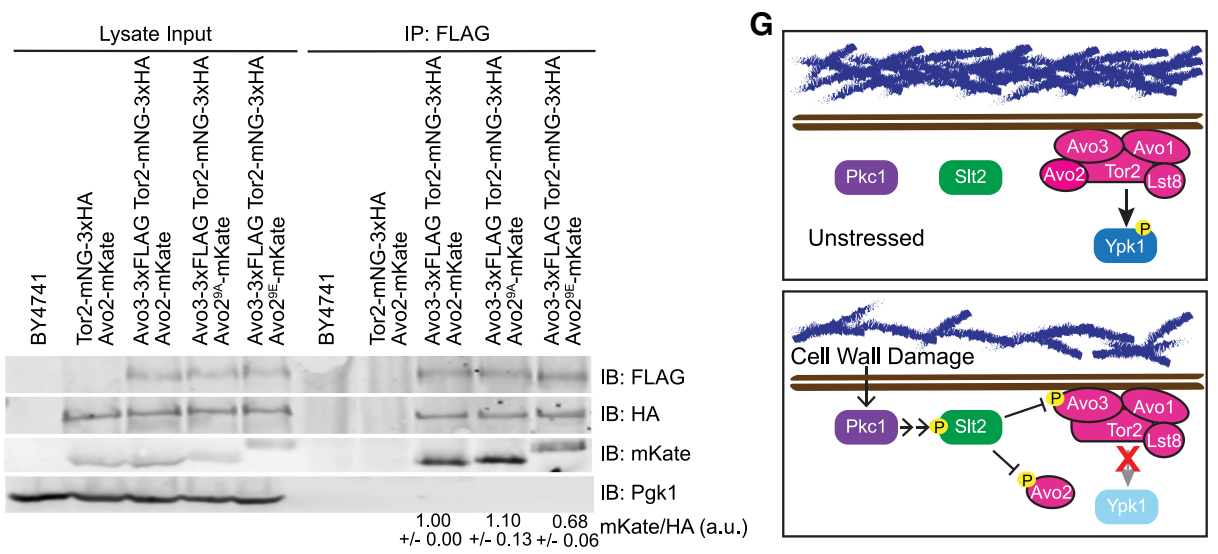

Figure 5. Phosphorylation at its MAPK sites displaces Avo2 from the PM. (A) Strains yAEA348 (Avo2 ${ }^{\mathrm{WT}}$-mNG-3xHA Pill-RFP), yAEA349 (Avo2 ${ }^{9 \mathrm{~A}}$-mNG-3xHA Pill-RFP), and yAEA350 (Avo2 ${ }^{9 \mathrm{E}}$-mNG-3xHA Pill-RFP) were grown to mid-exponential phase and examined by fluorescence microscopy as described in the Materials and Methods and processed using CellProfiler (as in Supplemental Fig. S3). (B) Mean values of the relative pixel intensities of the Pill-RFP and Avo2-mNG foci in images, as in $A$, were measured using CellProfiler, and those obtained for strain yAEA348 (Avo2 ${ }^{\mathrm{WT}}$-mNG-3xHA Pill-RFP) were set to $100 \%$, to which all of the other values were normalized. Error bars represent $95 \%$ confidence intervals. $\left.{ }^{* *}\right) P<0.0001 .(C)$ The same cells as in $A$ were resolved by standard SDS-PAGE and analyzed by immunoblotting. A representative experiment is shown. Values below the lanes represent the relative Avo2 to Pgk1 ratio (average of three independent experiments with SEM). (D) The mean pixel intensity of the Avo2-mNG foci that colocalized with PillRFP was measured using CellProfiler as in $B .\left(^{* *}\right) P<0.0001$. (E) The mean pixel intensity of the Avo2-mNG foci that did not colocalize with Pill-RFP was measured using CellProfiler as in $\left.B .{ }^{* *}\right) P<0.0001$. $(F)$ Strains BY4741, YFR589 (Avo2-mKate Tor2-mNG-3xHA), YFR624 (Avo2-mKate Avo3-3xFlag Tor2-mNG-3xHA), YFR626 (Avo2 ${ }^{9 \mathrm{~A}}$-mKate Avo3-3xFlag Tor2-mNG-3xHA), and YFR628 (Avo2 ${ }^{\text {9E }}$ mKate Avo3-3xFlag Tor2-mNG-3xHA) were propagated in YPD, harvested, and lysed, and equal amounts of the resulting extracts were immunoprecipitated with anti-Flag antibody. Proteins in the input extracts and in the immunoprecipitates were resolved by SDS-PAGE and analyzed by immunoblotting. A representative experiment is shown. Values below the right lanes represent the relative Avo2 to Tor2 ratio (average of three independent experiments with SEM), with the value obtained for strain YFR624 (Avo2-mKate Avo3$3 x$ Flag Tor2-mNG-3xHA) set as 1.00. (G) Model showing that under conditions that damage cell wall structure, activation of the CWI pathway exerts feedback that will negatively regulate the growth-promoting functions of TORC2.

phosphorylation of its primary downstream effector, AGC family kinase Ypk1 (mammalian ortholog SGK1) (Casamayor et al. 1999). We also found that modification of Avo2 was sufficient to explain Slt2-mediated inhibition of TORC2, but it is possible that Slt2 phosphorylation of Avo3 also contributes to TORC2 down-modulation. Likewise, all of the other subunits of TORC2 contain potential
MAPK phosphorylation sites; however, unlike for Avo2 and Avo3, phosphorylation at very few of these other candidate sites has been detected in global phosphoproteomic studies.

In any event, the control circuit that we discovered makes physiological sense because under conditions that interfere with cell wall synthesis or damaged cell 
wall structure, activation of the CWI pathway will exert feedback that will negatively regulate the growth-promoting functions of TORC2 (Fig. 5G). For yeast cell growth, enlargement of the cell wall needs to be tightly coupled to both an increase in cell mass and expansion of the PM. TORC2-Ypk1 signaling controls the processes that maintain adequate PM levels of all of the lipid classes (sphingolipids, glycerolipids, and sterols) needed to sustain growth and viability (Roelants et al. 2017a, 2018). Thus, down-regulation of TORC2 activity upon CWI pathway activation provides a mechanism by which the status of the cell wall can be sensed by TORC2 and thereby the rates of the reactions necessary for PM homeostasis can be adjusted accordingly.

The need for regulatory circuitry in which the state of the cell wall can feed in and influence TORC2 function perhaps explains why a recognizable Avo 2 homolog is present throughout the vast majority of the fungal cladefrom $S$. cerevisiae and its sensu stricto relatives to divergent yeast species (e.g., Debaromyces hansenii and Yarrowia lipolytica) to filamentous fungi (e.g., Aspergillus nidulans and Neurospora crassa) (see https://portals. broadinstitute.org/cgi-bin/regev/orthogroups/show orthogroup.cgi?orf=YMR068W)-but is not conserved in multicellular organisms that lack a cell wall. In this same regard, genetic evidence indicates that the Slt2 ortholog (Pmk1) in fission yeast (Schizosaccharomyces pombe) has a role in negatively regulating TORC2-mediated activation of the fission yeast Ypk1 ortholog (Gad8) (Cohen et al. 2014), providing further support for conservation of the overall mechanism that we describe here.

Our results show that Avo2 is not only an important site of Slt2-mediated phosphorylation but also necessary for optimal TORC2 activity, especially under stressful conditions. However, the function of Avo2 in TORC2 is not currently known. Within its N-terminal half, Avo2 contains at least five ankyrin repeats (Gaubitz et al. 2016), which are known to mediate protein-protein interactions but, in some cases, also interactions with other classes of biomolecules. Initial dissection of the subunit contacts in yeast TORC2 by coimmunoprecipitation and pull-down assays suggested that Avo2 associates only with Avol and Avo3 (Wullschleger et al. 2005). However, recent modeling of a predicted Avo2 structure into the cryo-electron microscopy (cryo-EM) density of yeast TORC2, if interpreted correctly, indicates intimate contact of Avo2 with the HEAT repeat and FAT domain regions of Tor2, much less extensive interaction with Avo3, and no contact with Avol (Karuppasamy et al. 2017). Nonetheless, this placement is surface-exposed and quite distant from the active site of the kinase domain in Tor2 and hence does not readily suggest an obvious function for Avo2 (Karuppasamy et al. 2017).

As first detected in global two-hybrid screens, Avo2 also interacts with two other paralogous proteins, $\operatorname{Slm} 1$ and $\operatorname{Slm} 2$, and the ability of Avo2 to associate with SIm1 and $\operatorname{Slm} 2$ also has been observed by both coimmunoprecipitation (Audhya et al. 2004) and in vitro binding assays (Fadri et al. 2005). Slm 1 and $S \operatorname{lm} 2$ are components of the furrowlike PM invaginations called eisosomes that are coated with the PtdIns4,5 $\mathrm{P}_{2}$-binding F-BAR domain-containing proteins Pill and Lsp1 (Karotki et al. 2011). Similarly, the central $\sim 190$ residues of Slm1 (686 residues) and Slm2 (656 residues) contain a predicted I-BAR (inverse $\mathrm{BAR}$ ) domain (which is required for their targeting to eisosomes) (Olivera-Couto et al. 2011) and a demonstrated 110-residue PtdIns4,5 $\mathrm{P}_{2}$-binding $\mathrm{PH}$ domain near their C-terminal ends (Audhya et al. 2004; Gallego et al. 2010). Absence of $S \operatorname{lm} 1$ and $S \operatorname{lm} 2$ reportedly disrupts eisosome structure (Kamble et al. 2011).

It has been observed before that each component subunit of TORC2 localizes around the cell periphery in multiple PM-associated "dots" and that these puncta are quite dynamic (Berchtold and Walther 2009), whereas the eisosomes are considered rather static structures (Brach et al. 2011). Moreover, it had been suggested that these two compartments are discrete (Berchtold and Walther 2009|. However, as we found and document here, a substantial fraction of Avo2 is readily found in association with the diagnostic eisosome marker Pill, in agreement with the observations of at least one other group (Bartlett et al. 2015). Furthermore, there is convincing evidence that the Avo2-interacting proteins and eisosome components $\operatorname{Slm} 1$ and $\operatorname{Slm} 2$ are required for TORC2-mediated phosphorylation of Ypk1 (Berchtold et al. 2012; Niles et al. 2012). However, the model proposed was that, under appropriate stress conditions (including hypotonic shock and sphingolipid depletion), $\operatorname{Slm} 1 / 2$ dissociate from eisosomes, bind Ypk1, and deliver this substrate to the TORC2 compartment (Berchtold et al. 2012; Niles et al. 2012). On the other hand, equally compelling data demonstrate that it is the CRIM element present in Avol and its orthologs in fission yeast (Sin1) and mammalian cells (mSIN1) that binds and presents substrates to the catalytic center in TORC2 (Liao and Chen 2012; Tatebe and Shiozaki 2017).

Hence, the findings that we describe here suggest an obverse model; namely, that the role of Avo2 (assuming, as its gene name indicates, that it "associates voraciously" with Tor2 and the other components of TORC2) is to act as an adaptor that, through its association with $\operatorname{Slm} 1 / \operatorname{Slm} 2$, is responsible for the dynamic docking of TORC2 at eisosomes. What, then, is the function of the Avo2-Slm1/2 interaction in promoting TORC2 function? As demonstrated by multiple groups (Bultynck et al. 2006; Tabuchi et al. 2006), Slm1/2 also bind tightly $\mathrm{Ca}^{+2} / \mathrm{cal}$ modulin-activated phosphoprotein phosphatase 2B (also known as calcineurin) and do so via canonical so-called PxIxIT motifs located very near their C termini $\left({ }^{673}\right.$ PNIYIQ $^{678}$ in Slm 1 and ${ }^{640}$ PEFYIE $^{645}$ in Slm2). Hence, it is possible that the calcineurin bound to $\operatorname{Slm} 1 / 2$ acts to remove the inhibitory phosphorylations present on Avo2 and other TORC2 subunits, thereby alleviating this negative regulation. Consistent with this model, we found that Avo $2^{9 \mathrm{E}}$, an allele that mimics the permanently phosphorylated state and hence would be immune to calcineurin action, was inhibitory to optimal TORC2 function in vivo, exhibited enhanced dissociation from PM sites, and reduced association with TORC2. Thus, based on the behavior of this phosphomimetic allele, a primary 
effect of Slt2-mediated phosphorylation is to impede its integration into TORC2 and interfere with Avo2-mediated association of TORC2 with the cell cortex. Whether Slt2 phosphorylation of Avo3 (or other TORC2 subunits) further promotes this dissociation and other ramifications of this model provide fertile ground for further study.

Finally, what we reveal here is one molecular mechanism by which a stress signal restrains a growth-promoting signal. As we document here for yeast, it seems likely that MAPK pathways activated by stress will impede TORC2 function in mammalian cells in a similar manner because continued growth and response to stress are invariably incompatible cellular states.

\section{Materials and methods}

\section{Construction of yeast strains and growth conditions}

Unless indicated otherwise in Table 1, S. cerevisiae strains used in this work were all constructed using conventional yeast genetic methods (Sherman 2002). Unless stated otherwise, yeast cultures were grown in standard rich $(\mathrm{YP})$ medium or defined minimal (SC) medium (Sherman 2002) containing 2\% glucose/ dextrose and were supplemented with the appropriate nutrients to permit growth of auxotrophs and/or select for plasmids. Cultures were propagated at $30^{\circ} \mathrm{C}$, unless indicated otherwise. Induction of genes under the control of GAL promoters was carried out either by adding $2 \%$ galactose to strains pregrown in the appropriate SC medium with $2 \%$ raffinose and $0.2 \%$ sucrose followed by incubation over the course of $3 \mathrm{~h}$ or, for strains containing the Gal4 human estrogen receptor herpes simplex virus transactivator VP16 fusion protein (Gal4-ER-VP16 [GEV]) (Quintero et al. 2007 ), by addition of $20 \mu \mathrm{M} \beta$-estradiol (final concentration) followed by incubation over the course of $3 \mathrm{~h}$. For strains containing a protein tagged with an AID, the cells were cultured in YPD or the appropriate SC medium buffered with $50 \mathrm{mM}$ potassium phosphate (pH 6.2), and degradation was induced by addition of a synthetic auxin (1-NAA) (Sigma-Aldrich) for the indicated time periods.

\section{Plasmids and recombinant DNA methods}

Plasmids used in this work (Table 2) were constructed using standard procedures in Escherichia coli strain DH5a (Green and Sambrook 2012). All PCR reactions were performed with Phusion high-fidelity DNA polymerase (New England Biolabs, Inc.). Site-directed mutagenesis was performed by using the appropriate mismatch oligonucleotide primers with the QuikChange method (Agilent Technologies, Inc.) according to the manufacturer's instructions. The fidelity of all constructs was verified by nucleotide sequence analysis.

\section{Cell extract preparation and immunoblotting}

Samples of exponentially-growing cells were harvested by brief centrifugation and stored at $-80^{\circ} \mathrm{C}$. Cell pellets were thawed on ice and lysed in $150 \mu \mathrm{L}$ of $1.85 \mathrm{M} \mathrm{NaOH}$ and $7.4 \% \beta$-mercaptoethanol. Proteins were precipitated by the addition of $150 \mu \mathrm{L}$ of $50 \%$ trichloroacetic acid for $10 \mathrm{~min}$ on ice. Precipitated proteins were pelleted by centrifugation and washed twice with ice-cold acetone. Protein pellets were solubilized in 5\% SDS in $0.1 \mathrm{M}$ Tris base, adjusted to a final concentration of $0.025 \mathrm{~A}_{600} \mathrm{~nm}$ per microliter, mixed with one-fifth volume of $5 \times$ SDS sample buffer, boiled for $10 \mathrm{~min}$, and then resolved by SDS-PAGE. Phospho- proteins were resolved by Phos-tag SDS-PAGE (Kinoshita et al. 2009): Phosphorylated Ypk1-myc was resolved in $8 \%$ acrylamide gels containing $35 \mu \mathrm{M}$ Phos-tag affinity reagent (Wako Chemicals USA, Inc.) and $70 \mu \mathrm{M} \mathrm{MnCl}{ }_{2}$, phosphorylated Avo2-3xFlag was resolved by in $8 \%$ acrylamide gels containing $17.5 \mu \mathrm{M}$ Phos-tag reagent and $35 \mu \mathrm{M} \mathrm{MnCl}_{2}$, and phosphorylated Avo3-3xFlag was resolved in $8 \%$ acrylamide gels containing $20 \mu \mathrm{M}$ Phos-tag reagent and $40 \mu \mathrm{M} \mathrm{MnCl}_{2}$.

For immunoblotting, proteins in gels were transferred electrophoretically to a nitrocellulose membrane, incubated with Odyssey blocking buffer (Li-Cor Biosciences, Inc.) diluted 1:1 with PBS or TBS, and then probed by addition of the appropriate primary antibody at the indicated dilution: mouse anti-myc mAb 9E10 (1:100; Monoclonal Antibody Facility, Cancer Research Laboratory, University of California at Berkeley), mouse anti-Flag M2 mAb (1:10,000; Sigma-Aldrich), mouse anti-HA.11 mAb (1:1000; BioLegend), rabbit polyclonal anti-RFP (1:5000; Rockland Immunochemicals), rabbit polyclonal anti-tRFP (1:1000 to detect mKate; Evrogen), or rabbit polyclonal anti-Pgk1 (1:30,000; this laboratory, prepared as described in Baum et al. 1978). Activated (dually phosphorylated) Hog1 was detected using rabbit anti-p38 MAPK phospho-Thr180/phospho-Tyr182 mAb (1:1000; Cell Signaling Technology), and activated (dually phosphorylated) Slt2 was detected using rabbit anti-p44/42 (Erk1/2) MAPK phospho-Thr202/phospho-Tyr204 mAb (1:1000; Cell Signaling Technology). After rinsing the membranes, filter-bound immune complexes were detected with an appropriate infrared dye-labeled secondary antibody-CF770-conjugated goat anti-mouse IgG (Biotium), IRDye800CW-conjugated goat anti-rabbit IgG (LiCor), or IRDye680RD-conjugated goat anti-mouse IgG (Li-Cor) -that was diluted 1:10,000 in 1:1 Odyssey blocking buffer::PBS (or TBS) containing $0.1 \%$ Tween- 20 and $0.02 \%$ SDS and visualized using an infrared imaging system (Odyssey CLx, Li-Cor).

S1t2 in vitro kinase assay

To purify activated Slt2, $1 \mathrm{~L}$ each of yeast strain CGA84 cotransformed with $\mathrm{P}_{G A L 1}-P K C 1^{*}$ and either pKL63 (P $\mathrm{P}_{G A L 1}$-Slt2 ${ }^{\mathrm{WT}}$-TAP) or pKL64 $\left(\mathrm{P}_{\text {GAL1 }}-\mathrm{Slt} 2^{\mathrm{KD}}\right.$-TAP) was grown to mid-exponential phase at $30^{\circ} \mathrm{C}$. Expression was induced by addition of $20 \mu \mathrm{M} \beta$ estradiol followed by incubation for $3 \mathrm{~h}$. The cells were harvested, washed once in ice-cold TAP-B buffer containing phosphatase and protease inhibitors $(200 \mathrm{mM} \mathrm{NaCl}, 1.5 \mathrm{mM}$ MgOAc, $1 \mathrm{mM}$ DTT, $2 \mathrm{mM} \mathrm{NaVO}_{4}, 10 \mathrm{mM} \mathrm{NaF}, 10 \mathrm{mM} \mathrm{Na}-\mathrm{PPi}, 10 \mathrm{mM} \beta$ glycerol phosphate, $1 \times$ complete protease inhibitor [Roche], $50 \mathrm{mM}$ Tris- $\mathrm{HCl}$ at $\mathrm{pH} 7.5$ ), resuspended in $4 \mathrm{~mL}$ of TAP-B buffer, and flash-frozen as droplets in liquid $\mathrm{N}_{2}$. The cells were ruptured cryogenically in a Mixer Mill MM301 (Retsch). The resulting lysate was thawed on ice, diluted with $8 \mathrm{~mL}$ of TAP-B buffer, and clarified by centrifugation at $15,000 \mathrm{~g}$ for $20 \mathrm{~min}$. The resulting clarified extract was subjected to centrifugation at 78,000 $\mathrm{g}$ for 1 $\mathrm{h}$, and the resulting supernatant fraction was adjusted to a final concentration of $0.15 \%$ NP-40 using an appropriate volume of a $10 \%$ NP-40 stock. Slt2-TAP in the detergent-containing soluble fraction was captured by binding to IgG-agarose resin (GE Healthcare). After washing the resin extensively with TAP wash buffer (200 mM NaCl, $1.5 \mathrm{mM} \mathrm{MgOAc,} 1 \mathrm{mM}$ DTT, 0.01\% NP-40, $2 \mathrm{mM} \mathrm{NaVO}_{4}, 10 \mathrm{mM} \mathrm{NaF}, 10 \mathrm{mM} \mathrm{Na}-\mathrm{PPi}, 10 \mathrm{mM} \beta$-glycerol phosphate, $50 \mathrm{mM}$ Tris- $\mathrm{HCl}$ at $\mathrm{pH} 7.5$ ), it was washed with Protease $3 \mathrm{C}$ buffer $(200 \mathrm{mM} \mathrm{NaCl}, 1.5 \mathrm{mM} \mathrm{MgOAc}, 1 \mathrm{mMDTT}, 0.01 \%$ NP-40, $10 \%$ glycerol, $2 \mathrm{mM} \mathrm{NaVO}_{4}, 10 \mathrm{mM} \mathrm{NaF}, 10 \mathrm{mM} \mathrm{Na}-\mathrm{PPi}$, $10 \mathrm{mM} \beta$-glycerol phosphate, $50 \mathrm{mM}$ Tris- $\mathrm{HCl}$ at $\mathrm{pH} 7.5)$. To elute the bound Slt2-TAP, the washed resin was resuspended in $500 \mu \mathrm{L}$ of Protease 3C buffer containing $60 \mathrm{U}$ of PreScission protease (GE Healthcare). After incubation for $5 \mathrm{~h}$ at $4^{\circ} \mathrm{C}$, the resin was 
Table 1. S. cerevisiae strains used in this study

\begin{tabular}{|c|c|c|}
\hline Strain & Genotype & Source or reference \\
\hline BY4741 & 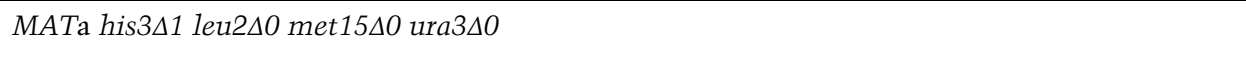 & $\begin{array}{l}\text { Research Genetics, } \\
\text { Inc. }\end{array}$ \\
\hline BY4742 & MATa his3 $\Delta 1$ leu2 $\Delta 0$ lys2 $2 \Delta 0$ ura3 $\Delta 0$ & $\begin{array}{l}\text { Research Genetics, } \\
\text { Inc. }\end{array}$ \\
\hline JTY5336 & BY4741 trp1::URA3 & This study \\
\hline YFR549 & BY4741 slt2 $2::$ KanMX trp1::URA3 & This study \\
\hline YFR538-A & BY4742 hog1 $1:: \operatorname{Kan} M X \operatorname{trp} 1:: U R A 3$ & This study \\
\hline YFR560 & BY4741 kss1A::CgHIS3 trp1::URA3 & This study \\
\hline YFR559 & BY4741 slt2 $::$ KanMX hog1 $1 \Delta:: \operatorname{KanMX} \operatorname{trp} 1:: U R A 3$ & This study \\
\hline YFR567 & 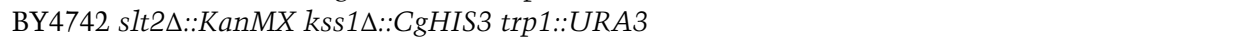 & This study \\
\hline YFR564 & 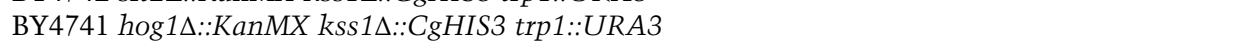 & This study \\
\hline YFR617 & BY4741 Avo3-3xFlag::KanMX trp1::URA3 & This study \\
\hline JTY5473 & BY4741 sln1ts-4::KanMX & $\begin{array}{l}\text { Costanzo et al. } \\
2010\end{array}$ \\
\hline yKL15 & BY4741 Sln1-6xHA::HygR TIR1::HIS3 & This study \\
\hline yKL18 & BY4741 Sln1-AID*-6xHA::HygR TIR1::HIS3 & This study \\
\hline yKL20 & BY4741 lys2 $\Delta 0$ Sln1-AID*-6xHA::HygR hog1 $1:: \operatorname{KanMX}$ TIR1::HIS3 & This study \\
\hline yKL16 & BY4741 Sln1-AID*-6xHA slt2A::URA3 TIR1::HIS3 & This study \\
\hline yKL22 & 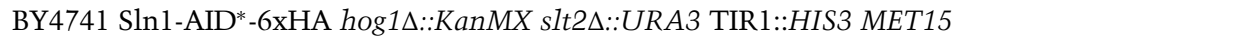 & This study \\
\hline JTY7318 & BY4742 avo2 $\Delta::$ KanMX & $\begin{array}{l}\text { Research Genetics, } \\
\text { Inc. }\end{array}$ \\
\hline yKL32 & BY4742 Avo2-3xFlag::URA3 & This study \\
\hline YFR181 & BY4742 Pill-RFP LYS2 met15AO & This study \\
\hline YFR528 & BY4742 Avo2(T144A T219A S233A S240A S249A T310A S315A T330A S333A)::URA3 & This study \\
\hline yKL31 & BY4742 Avo2(T144E T219E S233E S240E S249E T310E S315E T330E S333E)::URA3 & This study \\
\hline yKL33 & BY4742 Avo2(T144A T219A S233A S240A S249A T310A S315A T330A S333A)-3xFlag::URA3 & This study \\
\hline yKL34 & BY4742 Avo2(T144E T219E S233E S240E S249E T310E S315E T330E S333E)-3xFlag::URA3 & This study \\
\hline yEPS2 & BY4741 Pil1(S163D S230D T233D::URA3 & This study \\
\hline YFR641 & $\begin{array}{l}\text { BY4741 Avo2(T144E T219E S233E S240E S249E T310E S315E T330E S333E)::URA3 Pil1(S163D } \\
\text { S230D T233D::URA3 }\end{array}$ & This study \\
\hline yAEA348 & BY4741 Pill-RFP Avo2-mNG-3xHA::URA3 & This study \\
\hline yAEA349 & $\begin{array}{l}\text { BY4741 Pil1-RFP Avo2(T144A T219A S233A S240A S249A T310A S315A T330A S333A)-mNG- } \\
\text { 3xHA::URA3 }\end{array}$ & This study \\
\hline yAEA350 & $\begin{array}{l}\text { BY4741 Pill-RFP Avo2(T144E T219E S233E S240E S249E T310E S315E T330E S333E)-mNG- } \\
\text { 3xHA::URA3 }\end{array}$ & This study \\
\hline $\begin{array}{l}\text { yAEA301- } \\
\text { A }^{\mathrm{a}}\end{array}$ & BY4742 Tor2-mNG-3xHA & This study \\
\hline YFR589 & BY4741 Avo2-mKate::SpHIS5 Tor2-mNG-3xHA & This study \\
\hline YFR624 & BY4741 Avo2-mKate::SpHIS5 Tor2-mNG-3xHA Avo3-3xFlag::KanMX & This study \\
\hline YFR626 & $\begin{array}{l}\text { BY4741 Avo2(T144A T219A S233A S240A S249A T310A S315A T330A S333A)-mKate::SpHIS5 } \\
\text { Tor2-mNG-3xHA Avo3-3xFlag::KanMX }\end{array}$ & This study \\
\hline YFR628 & $\begin{array}{l}\text { BY4741 Avo2(T144E T219E S233E S240E S249E T310E S315E T330E S333E)-mKate::SpHIS5 } \\
\text { Tor2-mNG-3xHA Avo3-3xFlag::KanMX }\end{array}$ & This study \\
\hline CGA84 & 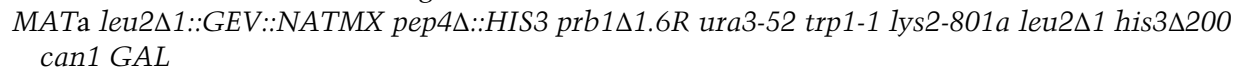 & Alvaro et al. 2014 \\
\hline
\end{tabular}

${ }^{a}$ For strain yAEA301-A, the coding sequence for an mNG-3xHA cassette was inserted in-frame between Asn321 and Thr322 of the TOR2 ORF on chromosome XI, constructed using a CRISPR-Cas9-based method. In brief, BY4742 was transformed with URA3marked pRS316-GAL prom -Cas9 (pGF-V789) (Finnigan and Thorner 2016), expression of Cas9 was induced in a resulting transformant by addition of galactose ( $\%$ final concentration), and, after $6 \mathrm{~h}$, the cells were cotransformed with HIS3-marked pRS423-sgRNA-Tor2 (pAEA272) and a TOR2-mNG-3xHA-TOR2 repair cassette generated and amplified by overlap extension PCR using appropriate DNA templates and synthetic oligonucleotide primers. The desired (viable) transformants were selected on SCD - Ura - His plates and then colony-purified by streaking on SD + Ura + His plates containing 5-FOA (to select for derivatives that lost pGF-V789 and pAEA272). The single-guide RNA (sgRNA) encoded by pRS423-sgRNA-Tor2 (pAEA272) was ${ }^{-463}$ ATTTTGGCGCCAGTGA TAAGTGG $^{-441}$ (the PAM sequence is underlined) directed against the corresponding sequence within the TOR2 promoter (where +1 is the first base of the initiator ATG of the TOR2 ORF). In the upstream primer used to generate the TOR2-mNG-3xHA-TOR2 repair cassette DNA, to prevent recognition by the sgRNA, the sequence in and around the PAM was mutated to ${ }^{-446}$ TAGTCG $^{-441}$. The proper in-frame insertion of the mNG-3xHA-coding sequence in the TOR2 ORF was verified by colony PCR and DNA sequencing.

removed by centrifugation, and the Protease $3 \mathrm{C}$ in the resulting supernatant faction was removed by incubation with a slurry of glutathione-Sepharose beads (GE Healthcare) followed by centri- fugation. The affinity-purified Slt2 in the resulting final supernatant solution was diluted into kinase assay buffer $(125 \mathrm{mM}$ potassium acetate, $5 \%$ glycerol, $12 \mathrm{mM} \mathrm{MgCl}_{2}, 0.5 \mathrm{mM}$ EGTA, 
Table 2. Plasmids used in this study

\begin{tabular}{|c|c|c|}
\hline Plasmid & Description & Source or reference \\
\hline pRS315 & CEN, LEU2, vector & $\begin{array}{l}\text { Sikorski and Hieter } \\
1989\end{array}$ \\
\hline pFR246 & pRS315 Ypk1(T51A T71A T504A S644A T662A)-myc & Leskoske et al. 2017 \\
\hline pKL1 & pRS315 Avo2-3xFlag & This study \\
\hline pKL2 & $\begin{array}{l}\text { pRS315 Avo2(T144A T219A S233A S240A S249A T310A S315A T330A S333A)- } \\
\text { 3xFlag }\end{array}$ & This study \\
\hline pFR234 & pRS315 Ypk1(D242A)-myc & Roelants et al. $2017 \mathrm{~b}$ \\
\hline yEPlac112 & $2 \mu \mathrm{m}, \mathrm{TRP} 1$, vector & Gietz and Sugino 1988 \\
\hline $\mathrm{Pkc1}^{*}(\mathrm{pJT} 5660)$ & YEplac112 $\mathrm{P}_{G A L 1}-\mathrm{Pkc1}(\mathrm{R} 398 \mathrm{~A}$ R405A R406A) & Martín et al. 2000 \\
\hline $\begin{array}{l}\mathrm{pJEN} 12 \\
\quad\left(\mathrm{Pkcl}^{*} \mathrm{KD}\right)\end{array}$ & YEplac112 $\mathrm{P}_{G A L 1}$-Pkc1(R398A R405A R406A D949A) & This study \\
\hline pAEA376 & YEplac112 $\mathrm{P}_{G A L 1-\mathrm{Pkc1}(\mathrm{R} 398 A}$ R405A R406A)-myc & This study \\
\hline pJEN13 & YEplac112 $\mathrm{P}_{\text {GAL1 }}$-Pkc1(R398A R405A R406A D949A)-myc & This study \\
\hline BG1805 & $2 \mu \mathrm{m}, U R A 3, \mathrm{P}_{G A L 1}, \mathrm{C}$-terminal tandem affinity (TAP) tag vector & Open Biosystems, Inc. \\
\hline pKL63 & BG1805 Slt2 & This study \\
\hline pKL64 & BG1805 Slt2(K54R) & This study \\
\hline pGEX6P-1 & GST tag, bacterial expression vector & GE Healthcare, Inc. \\
\hline pKL16 & pGEX6P-1 Avo2 & This study \\
\hline pKL17 & pGEX6P-1 Avo2(T144A T219A S233A S240A S249A T310A S315A T330A S333A) & This study \\
\hline pKL81 & pGEX6P-1 Avo3(1-100) & This study \\
\hline pKL82 & pGEX6P-1 Avo3(1-100)(S11A T24A T28A S50A S84A S87A) & This study \\
\hline
\end{tabular}

$2 \mathrm{mM}$ DTT, $1 \mathrm{mM}$ PMSF, $0.1 \mathrm{mM}$ chymostatin, $4 \mathrm{mM}$ AEBSF, $12.5 \mathrm{mM} \beta$-glycerol phosphate, $1 \mathrm{mM} \mathrm{NaVO}_{4}, 40 \mathrm{mM}$ Tris-HCl at $\mathrm{pH} 7.5$.

Substrates to be tested were purified as recombinant proteins by fusion to the $\mathrm{C}$ terminus of GST in appropriate pGEX vectors and expressed in E. coli BL21(DE3)pLysS. One liter of cultures was grown at $30^{\circ} \mathrm{C}$ to mid-exponential phase and then induced with $0.5 \mathrm{mM}$ IPTG for $3 \mathrm{~h}$ at $30^{\circ} \mathrm{C}$. The bacterial cells were harvested by centrifugation and lysed, and the GST fusion protein of interest was adsorbed to glutathione-agarose beads (Prometheus Laboratories, Inc.), which were washed and resuspended in kinase assay buffer. The same volume of either affinity-purified Slt2 ${ }^{\text {WT }}$ or Slt $2^{\mathrm{KD}}$ was added to the resuspended beads or an equivalent volume of kinase assay buffer, and reaction was initiated by the addition of $2 \mu \mathrm{Ci}$ of $\left[\gamma^{-32} \mathrm{P}\right] \mathrm{ATP}$ and unlabeled ATP to $100 \mu \mathrm{M}$ final concentration. After incubation for $30 \mathrm{~min}$ at $30^{\circ} \mathrm{C}$, reactions were terminated by addition of $5 \times$ SDS-PAGE sample buffer followed by boiling for $10 \mathrm{~min}$. The resulting reaction products were resolved by SDS-PAGE and analyzed by Coomassie blue staining and autoradiography using a phosphorimager (Typhoon, GE Healthcare).

\section{Immunoprecipitation of TORC2}

One-hundred milliliters of cultures of yeast strains expressing Avo3-3xFlag Tor2-mNG-3xHA and either Avo2-mKate (YFR624), Avo2 ${ }^{9 \mathrm{~A}}$-mKate (YFR626), or Avo2 ${ }^{9 \mathrm{E}}$-mKate (YFR628) was grown in YPD to mid-exponential phase, harvested by centrifugation, and frozen in liquid nitrogen. Cells pellets were resuspended in $500 \mu \mathrm{L}$ of $2 \times$ TNEG buffer $(100 \mathrm{mM}$ Tris at $\mathrm{pH} 7.6$, $300 \mathrm{mM} \mathrm{NaCl}, 20 \%$ glycerol, $0.24 \%$ Tergitol, $2 \mathrm{mM}$ EDTA, $0.1 \mathrm{mM}$ PMSF, $1 \times$ Roche complete protease inhibitor tablet [Roche]). Glass beads were added to the meniscus of the cell suspension, and lysis was achieved by vigorous vortex mixing for 3 min. After lysis, $2 \times$ vol of $1 \times$ TNEG buffer was added, and then the lysate was clarified by centrifugation at $1000 \mathrm{~g}$ for $5 \mathrm{~min}$ at $4^{\circ} \mathrm{C}$ followed by a second centrifugation at $2000 \mathrm{~g}$ for $10 \mathrm{~min}$. TORC 2 complexes marked by the tightly bound Avo3-3xFlag subunit were collected by immunoabsorption to $40 \mu \mathrm{L}$ of mouse anti-Flag affinity gel (Biotool) equilibrated in 1× TNEG buffer. The resin was washed four times with $1 \mathrm{~mL}$ of $1 \times$ TNEG buffer and resuspended in $2 \times$ urea buffer.

Fluorescence microscopy and image analysis

Fluorescence microscopy was performed using an Elyra PS.1 structured illumination microscope (Carl Zeiss AG) equipped with a $100 \times$ plan-apo 1.46 NA TIRF objective, a main focus drive of the AxioObserver Z1 stand, and a WSB PiezoDrive 08 controlled by Zen, and images were recorded using a $512 \times 512$ (100-nm $\times 100-n m$ pixel size) EM-CCD camera (Andor Technology). To visualize Avo 2 or Tor2 tagged with $\mathrm{mNG}$ (excitation $\lambda_{\max }$ $506 \mathrm{~nm}$; emission $\lambda_{\max } 517$ ), cell samples were excited with an argon laser at $488 \mathrm{~nm}$ at $2.3 \%$ power $(100 \mathrm{~mW})$, and emission was captured in a 495- to 550-nm window using a bandpass filter; for Pill tagged with RFP/DsRed (excitation $\lambda_{\max } 558 \mathrm{~nm}$; emission $\lambda_{\max } 583$ ), excitation was at $561 \mathrm{~nm}$ at $2.3 \%$ power (100 $\mathrm{mW}$ ), and emission was monitored in a 570- to 620-nm window using a different bandpass filter. Images (average of eight scans; $300 \mathrm{msec} / \mathrm{scan}$ ) were analyzed using Fiji (Schindelin et al. 2012). To avoid changes in image quality due to occasional fluctuations in laser intensity, all panels shown in any given figure represent experiments performed on the same day and scaled and adjusted identically for brightness using Fiji (Schindelin et al. 2012). For quantitative automated analysis of fluorescence intensity at the PM, CellProfiler was used (Carpenter et al. 2006). To train CellProfiler to apply the appropriate mask and quantify the signal, a corresponding pipeline was created, which was adapted from prior software (Bray et al. 2015; Chong et al. 2015). Prior to loading into the CellProfiler pipeline, cell images were segmented manually using Fiji (Schindelin et al. 2012). To avoid any selection bias, every cell visible in the bright-field image in a frame from any sample (except those out of focus) was chosen. All plots and statistical analyses in this study were performed with the $\mathrm{R}$ statistical analysis package (http://www. R-project.org). 


\section{Acknowledgments}

We thank Victor J. Cid and Maria Molina (University of Madrid Complutense) for the $\mathrm{Pkcl}^{*}$ plasmid, Steve Ruzin and Denise Schichnes (Biological Imaging Facility, University of California at Berkeley) for their invaluable advice about fluorescence microscopy, and all members of the Thorner laboratory for helpful discussions. We apologize in advance to any investigator whose work was not cited due to the limitation on the total number of allowed references imposed by the editorial policy of this journal. This work was supported by National Institutes of Health (NIH) Predoctoral Traineeship GM07232 and a University of California at Berkeley MacArthur and Lakhan-Pal Graduate Fellowship to K. L.L., Erwin Schroedinger Fellowship J3787-B21 from the Austrian Science Fund to A.E.-A., Marie Sklodowska-Curie Individual Fellowship GA 750835 from the European Commission to C.M.A., and NIH R01 research grant GM21841 to J.T. This work was also aided in part by NIH S10 Equipment Grant OD018136 (to Steven E. Ruzin, Director, University of California at Berkeley Biological Imaging Facility) for a Zeiss Elyra S1 structured illumination microscope.

Author contributions: K.L.L. and F.M.R. designed, executed, and analyzed experiments and drafted the manuscript. A.E.-A. designed, executed, and analyzed experiments and conducted fluorescence microscopy. C.M.A. set up the specific CellProfiler pipeline to quantify the fluorescent intensities and performed all of the statistical analyses. E.P.S. and J.M.H. provided technical assistance with several experiments. J.T. designed and analyzed experiments and revised the manuscript.

\section{References}

Alvaro CG, O'Donnell AF, Prosser DC, Augustine AA, Goldman A, Brodsky JL, Cyert MS, Wendland B, Thorner J. 2014. Specific a-arrestins negatively regulate Saccharomyces cerevisiae pheromone response by down-modulating the G-protein-coupled receptor Ste2. Mol Cell Biol 34: 2660-2681. doi:10.1128/ MCB.00230-14

Audhya A, Loewith R, Parsons AB, Gao L, Tabuchi M, Zhou H, Boone C, Hall MN, Emr SD. 2004. Genome-wide lethality screen identifies new PI4,5P2 effectors that regulate the actin cytoskeleton. EMBO J 23: 3747-3757. doi:10.1038/ sj.emboj. 7600384

Bartlett K, Gadila SK, Tenay B, McDermott H, Alcox B, Kim K. 2015. TORC2 and eisosomes are spatially interdependent, requiring optimal level of phosphatidylinositol 4, 5-bisphosphate for their integrity. I Biosci 40: 299-311. doi:10.1007/ s12038-015-9526-4

Baum P, Thorner J, Honig L. 1978. Identification of tubulin from the yeast Saccharomyces cerevisiae. Proc Natl Acad Sci 75: 4962-4966. doi:10.1073/pnas.75.10.4962

Berchtold D, Walther TC. 2009. TORC2 plasma membrane localization is essential for cell viability and restricted to a distinct domain. Mol Biol Cell 20: 1565-1575. doi:10.1091/mbc.e0810-1001

Berchtold D, Piccolis M, Chiaruttini N, Riezman I, Riezman H, Roux A, Walther TC, Loewith R. 2012. Plasma membrane stress induces relocalization of Slm proteins and activation of TORC2 to promote sphingolipid synthesis. Nat Cell Biol 14: 542-547. doi:10.1038/ncb2480

Brach T, Specht T, Kaksonen M. 2011. Reassessment of the role of plasma membrane domains in the regulation of vesicular traffic in yeast. I Cell Sci 124: 328-337. doi:10.1242/ jes.078519
Bray MA, Vokes MS, Carpenter AE. 2015. Using CellProfiler for automatic identification and measurement of biologicalobjects in images. Curr Protoc Mol Biol 109: 14.17.1-14.17.13. doi:10.1002/0471142727.mb1417s109

Bultynck G, Heath VL, Majeed AP, Galan JM, Haguenauer-Tsapis R, Cyert MS. 2006. Slm1 and Slm2 are novel substrates of the calcineurin phosphatase required for heat stress-induced endocytosis of the yeast uracil permease. Mol Cell Biol 26: 4729-4745. doi:10.1128/MCB.01973-05

Carpenter AE, Jones TR, Lamprecht MR, Clarke C, Kang IH, Friman O, Guertin DA, Chang JH, Lindquist RA, Moffat J, et al. 2006. CellProfiler: image analysis software for identifying and quantifying cell phenotypes. Genome Biol 7: R100. doi:10.1186/gb-2006-7-10-r100

Casamayor A, Torrance PD, Kobayashi T, Thorner J, Alessi DR. 1999. Functional counterparts of mammalian protein kinases PDK1 and SGK in budding yeast. Curr Biol 9: 186-197. doi:10.1016/S0960-9822/99/80088-8

Chong YT, Koh JL, Friesen H, Duffy SK, Duffy K, Cox MJ, Moses A, Moffat J, Boone C, Andrews BJ. 2015. Yeast proteome dynamics from single cell imaging and automated analysis. Cell 161: 1413-1424. doi:10.1016/j.cell.2015.04.051

Cohen A, Kupiec M, Weisman R. 2014. Glucose activates TORC2-Gad8 protein via positive regulation of the cAMP/ cAMP-dependent protein kinase A (PKA) pathway and negative regulation of the Pmk1 protein-mitogen-activated protein kinase pathway. J Biol Chem 289: 21727-21737. doi:10.1074/ jbc.M114.573824

Costanzo M, Baryshnikova A, Bellay J, Kim Y, Spear ED, Sevier CS, Ding H, Koh JL, Toufighi K, Mostafavi S, et al. 2010. The genetic landscape of a cell. Science 327: 425-431. doi:10.1126/science.1180823

Davenport KR, Sohaskey M, Kamada Y, Levin DE, Gustin MC. 1995. A second osmosensing signal transduction pathway in yeast. Hypotonic shock activates the $\mathrm{PKC1}$ protein kinaseregulated cell integrity pathway. I Biol Chem 270: 30157-30161. doi:10.1074/jbc.270.50.30157

de Nobel H, Ruiz C, Martin H, Morris W, Brul S, Molina M, Klis FM. 2000. Cell wall perturbation in yeast results in dual phosphorylation of the Slt2/Mpk1 MAP kinase and in an Slt2-mediated increase in FKS2-lacZ expression, glucanase resistance and thermotolerance. Microbiology 146: 2121-2132. doi:10.1099/00221287-146-9-2121

Douglas LM, Konopka JB. 2014. Fungal membrane organization: the eisosome concept. Annu Rev Microbiol 68: 377-393. doi:10.1146/annurev-micro-091313-103507

Fadri M, Daquinag A, Wang S, Xue T, Kunz J. 2005. The pleckstrin homology domain proteins $S \operatorname{lm} 1$ and $S \operatorname{lm} 2$ are required for actin cytoskeleton organization in yeast and bind phosphatidylinositol-4,5-bisphosphate and TORC2. Mol Biol Cell 16: 1883-1900. doi:10.1091/mbc.e04-07-0564

Finnigan GC, Thorner J. 2016. mCAL: a new approach for versatile multiplex action of Cas9 using one sgRNA and loci flanked by a programmed target sequence. G3 6: 2147-2156. doi:10.1534/g3.116.029801

Fradkov AF, Chen Y, Ding L, Barsova EV, Matz MV, Lukyanov SA. 2000. Novel fluorescent protein from Discosoma coral and its mutants possesses a unique far-red fluorescence. FEBS Lett 479: 127-130. doi:10.1016/S0014-5793(00)01895-0

Fuchs BB, Mylonakis E. 2009. Our paths might cross: the role of the fungal cell wall integrity pathway in stress response and cross talk with other stress response pathways. Eukaryot Cell 8: 1616-1625. doi:10.1128/EC.00193-09

Gallego O, Betts MJ, Gvozdenovic-Jeremic J, Maeda K, Matetzki C, Aguilar-Gurrieri C, Beltran-Alvarez P, Bonn S, Fernández- 
Tornero C, Jensen LJ, et al. 2010. A systematic screen for protein-lipid interactions in Saccharomyces cerevisiae. Mol Syst Biol 6: 430. doi:10.1038/msb.2010.87

García R, Bermejo C, Grau C, Pérez R, Rodríguez-Peña JM, Francois J, Nombela C, Arroyo J. 2004. The global transcriptional response to transient cell wall damage in Saccharomyces cerevisiae and its regulation by the cell integrity signaling pathway. I Biol Chem 279: 15183-15195. doi:10.1074/jbc. M312954200

García-Rodríguez LJ, Valle R, Durán A, Roncero C. 2005. Cell integrity signaling activation in response to hyperosmotic shock in yeast. FEBS Lett 579: 6186-6190. doi:10.1016/j. febslet.2005.10.001

García R, Rodríguez-Peña JM, Bermejo C, Nombela C, Arroyo J. 2009. The high osmotic response and cell wall integrity pathways cooperate to regulate transcriptional responses to zymolyase-induced cell wall stress in Saccharomyces cerevisiae. I Biol Chem 284: 10901-10911. doi:10.1074/jbc.M808693200

Gaubitz C, Prouteau M, Kusmider B, Loewith R. 2016. TORC2 structure and function. Trends Biochem Sci 41: 532-545. doi:10.1016/j.tibs.2016.04.001

Gietz RD, Sugino A. 1988. New yeast-Escherichia coli shuttle vectors constructed with in vitro mutagenized yeast genes lacking six-base pair restriction sites. Gene 74: 527-534. doi:10.1016/0378-1119(88)90185-0

González A, Hall MN. 2017. Nutrient sensing and TOR signaling in yeast and mammals. EMBO J 36: 397-408. doi:10.15252/ embj. 201696010

Green MR, Sambrook J. 2012. Molecular cloning: a laboratory manual. Cold Spring Harbor Laboratory Press, Cold Spring Harbor, NY.

Guerreiro JF, Muir A, Ramachandran S, Thorner J, Sá-Correia I. 2016. Sphingolipid biosynthesis upregulation by TOR complex 2-Ypk1 signaling during yeast adaptive response to acetic acid stress. Biochem I 473: 4311-4325. doi:10.1042/ BCJ20160565

Hahn JS, Thiele DJ. 2002. Regulation of the Saccharomyces cerevisiae Slt2 kinase pathway by the stress-inducible Sdp1 dual specificity phosphatase. I Biol Chem 277: 21278-21284. doi:10.1074/jbc.M202557200

Hatakeyama R, Kono K, Yoshida S. 2017. Ypk1 and Ypk2 kinases maintain Rhol at the plasma membrane by flippase-dependent lipid remodeling after membrane stresses. I Cell Sci 130: 1169-1178.

Helliwell SB, Schmidt A, Ohya Y, Hall MN. 1998. The Rho1 effector Pkc1, but not Bnil, mediates signalling from Tor2 to the actin cytoskeleton. Curr Biol 8: 1211-1214. doi:10.1016/ S0960-9822(07)00511-8

Hickman MJ, Spatt D, Winston F. 2011. The Hog1 mitogenactivated protein kinase mediates a hypoxic response in Saccharomyces cerevisiae. Genetics 188: 325-338. doi:10.1534/ genetics.111.128322

Kamada Y, Jung US, Piotrowski J, Levin DE. 1995. The protein kinase C-activated MAP kinase pathway of Saccharomyces cerevisiae mediates a novel aspect of the heat shock response. Genes Dev 9: 1559-1571. doi:10.1101/gad.9.13.1559

Kamada Y, Fujioka Y, Suzuki NN, Inagaki F, Wullschleger S, Loewith R, Hall MN, Ohsumi Y. 2005. Tor2 directly phosphorylates the AGC kinase Ypk2 to regulate actin polarization. Mol Cell Biol 25: 7239-7248. doi:10.1128/MCB.25.16.72397248.2005

Kamble C, Jain S, Murphy E, Kim K. 2011. Requirements of Slm proteins for proper eisosome organization, endocytic trafficking and recycling in the yeast Saccharomyces cerevisiae. J Biosci 36: 79-96. doi:10.1007/s12038-011-9018-0
Karotki L, Huiskonen JT, Stefan CJ, Ziółkowska NE, Roth R, Surma MA, Krogan NJ, Emr SD, Heuser J, Grünewald K, et al. 2011. Eisosome proteins assemble into a membrane scaffold. J Cell Biol 195: 889-902. doi:10.1083/jcb.201104040

Karuppasamy M, Kusmider B, Oliveira TM, Gaubitz C, Prouteau M, Loewith R, Schaffitzel C. 2017. Cryo-EM structure of Saccharomyces cerevisiae target of rapamycin complex 2. Nat Commun 8: 1729. doi:10.1038/s41467-017-01862-0

Kinoshita E, Kinoshita-Kikuta E, Koike T. 2009. Separation and detection of large phosphoproteins using Phos-tag SDSPAGE. Nat Protoc 4: 1513-1521. doi:10.1038/nprot.2009.154

Lawrence CL, Botting CH, Antrobus R, Coote PJ. 2004. Evidence of a new role for the high-osmolarity glycerol mitogen-activated protein kinase pathway in yeast: regulating adaptation to citric acid stress. Mol Cell Biol 24: 3307-3323. doi:10.1128/ MCB.24.8.3307-3323.2004

Lee YJ, Jeschke GR, Roelants FM, Thorner J, Turk BE. 2012. Reciprocal phosphorylation of yeast glycerol-3-phosphate dehydrogenases in adaptation to distinct types of stress. Mol Cell Biol 32: 4705-4717. doi:10.1128/MCB.00897-12

Leskoske KL, Roelants FM, Marshall MNM, Hill JM, Thorner J. 2017. The stress-sensing TORC2 complex activates yeast AGC-family protein kinase Ypk1 at multiple novel sites. Genetics 207: 179-195. doi:10.1534/genetics.117.1124

Levin DE. 2011. Regulation of cell wall biogenesis in Saccharomyces cerevisiae: the cell wall integrity signaling pathway. Genetics 189: 1145-1175. doi:10.1534/genetics.111.128264

Liao HC, Chen MY. 2012. Target of rapamycin complex 2 signals to downstream effector yeast protein kinase 2 (Ypk2) through adheres-voraciously-to-target-of-rapamycin-2 protein 1 (Avol) in Saccharomyces cerevisiae. I Biol Chem 287: 6089-6099. doi:10.1074/jbc.M111.303701

Martín H, Rodríguez-Pachón JM, Ruiz C, Nombela C, Molina M. 2000. Regulatory mechanisms for modulation of signaling through the cell integrity Slt2-mediated pathway in Saccharomyces cerevisiae. J Biol Chem 275: 1511-1519. doi:10.1074/ jbc.275.2.1511

Mascaraque V, Hernáez ML, Jiménez-Sánchez M, Hansen R, Gil C, Martín H, Cid VJ, Molina M. 2013. Phosphoproteomic analysis of protein kinase $\mathrm{C}$ signaling in Saccharomyces cerevisiae reveals Slt2 mitogen-activated protein kinase (MAPK)-dependent phosphorylation of eisosome core components. Mol Cell Proteomics 12: 557-574. doi:10.1074/mcp.M112.020438

Mok J, Kim PM, Lam HY, Piccirillo S, Zhou X, Jeschke GR, Sheridan DL, Parker SA, Desai V, Jwa M, et al. 2010. Deciphering protein kinase specificity through large-scale analysis of yeast phosphorylation site motifs. Sci Signal 3: ra12. doi:10.1126/ scisignal.2000482

Morawska M, Ulrich HD. 2013. An expanded tool kit for the auxin-inducible degron system in budding yeast. Yeast 30: 341-351. doi:10.1002/yea.2967

Muir A, Ramachandran S, Roelants FM, Timmons G, Thorner J. 2014. TORC2-dependent protein kinase Ypk1 phosphorylates ceramide synthase to stimulate synthesis of complex sphingolipids. Elife doi:10.7554/eLife.03779

Muir A, Roelants FM, Timmons G, Leskoske KL, Thorner J. 2015. Down-regulation of TORC2-Ypk1 signaling promotes MAPK-independent survival under hyperosmotic stress. Elife doi:10.7554/eLife.09336

Niles BJ, Powers T. 2014. TOR complex 2-Ypk1 signaling regulates actin polarization via reactive oxygen species. Mol Biol Cell 25: 3962-3972. doi:10.1091/mbc.e14-06-1122

Niles BJ, Mogri H, Hill A, Vlahakis A, Powers T. 2012. Plasma membrane recruitment and activation of the AGC kinase Ypk1 is mediated by target of rapamycin complex 2 
(TORC2) and its effector proteins Slm1 and Slm2. Proc Nat1 Acad Sci 109: 1536-1541. doi:10.1073/pnas.1117563109

Nomura W, Inoue Y. 2015. Methylglyoxal activates the target of rapamycin complex 2-protein kinase $\mathrm{C}$ signaling pathway in Saccharomyces cerevisiae. Mol Cell Biol 35: 1269-1280. doi:10.1128/MCB.01118-14

Olivera-Couto A, Graña M, Harispe L, Aguilar PS. 2011. The eisosome core is composed of BAR domain proteins. Mol Biol Cell 22: 2360-2372. doi:10.1091/mbc.e10-12-1021

Panadero J, Pallotti C, Rodríguez-Vargas S, Randez-Gil F, Prieto JA. 2006. A downshift in temperature activates the high osmolarity glycerol (HOG) pathway, which determines freeze tolerance in Saccharomyces cerevisiae. I Biol Chem 281: 4638-4645. doi:10.1074/jbc.M512736200

Perez P, Rincón SA. 2010. Rho GTPases: regulation of cell polarity and growth in yeasts. Biochem J 426: 243-253. doi:10.1042/ BJ20091823

Quintero MJ, Maya D, Arévalo-Rodríguez M, Cebolla Á, Chávez S. 2007. An improved system for estradiol-dependent regulation of gene expression in yeast. Microb Cell Fact 6: 10. doi:10.1186/1475-2859-6-10

Rodríguez-Peña JM, Díez-Muñiz S, Bermejo C, Nombela C, Arroyo J. 2013. Activation of the yeast cell wall integrity MAPK pathway by zymolyase depends on protease and glucanase activities and requires the mucin-like protein $\mathrm{Hkrl}$ but not Msb2. FEBS Lett 587: 3675-3680. doi:10.1016/j. febslet.2013.09.030

Roelants FM, Torrance PD, Bezman N, Thorner J. 2002. Pkh1 and Pkh2 differentially phosphorylate and activate Ypk1 and Ykr2 and define protein kinase modules required for maintenance of cell wall integrity. Mol Biol Cell 13: 3005-3028. doi:10.1091/mbc.e02-04-0201

Roelants FM, Breslow DK, Muir A, Weissman JS, Thorner J. 2011. Protein kinase Ypk1 phosphorylates regulatory proteins Orm 1 and Orm 2 to control sphingolipid homeostasis in Saccharomyces cerevisiae. Proc Natl Acad Sci 108: 19222-19227. doi:10.1073/pnas.1116948108

Roelants FM, Leskoske KL, Martinez Marshall MN, Locke MN, Thorner J. 2017a. The TORC2-dependent signaling network in the yeast Saccharomyces cerevisiae. Biomolecules 7: E66. doi:10.3390/biom7030066

Roelants FM, Leskoske KL, Pedersen RT, Muir A, Liu JM, Finnigan GC, Thorner J. 2017b. TOR complex 2-regulated protein kinase Fpk1 stimulates endocytosis via inhibition of Ark1/ Prk1-related protein kinase Akl1 in Saccharomyces cerevisiae. Mol Cell Biol 37: e00627-16. doi:10.1128/MCB.00627-16

Roelants FM, Chauhan N, Muir A, Davis JC, Menon AK, Levine TP, Thorner J. 2018. TOR Complex 2-regulated protein kinase Ypk1 controls sterol distribution by inhibiting StARkin domain-containing proteins located at plasma membrane-endoplasmic reticulum contact sites. Mol Biol Cell 29: 2128-2136. doi:10.1091/mbc.E18-04-0229

Roux PP, Blenis J. 2004. ERK and p38 MAPK-activated protein kinases: a family of protein kinases with diverse biological functions. Microbiol Mol Biol Rev 68: 320-344. doi:10.1128/ MMBR.68.2.320-344.2004

Saito H, Posas F. 2012. Response to hyperosmotic stress. Genetics 192: 289-318. doi:10.1534/genetics.112.140863

Schindelin J, Arganda-Carreras I, Frise E, Kaynig V, Longair M, Pietzsch T, Preibisch S, Rueden C, Saalfeld S, Schmid B, et al. 2012. Fiji: an open-source platform for biological-image analysis. Nat Methods 9: 676-682. doi:10.1038/nmeth.2019

Schmidt A, Kunz J, Hall MN. 1996. TOR2 is required for organization of the actin cytoskeleton in yeast. Proc Natl Acad Sci 93: 13780-13785. doi:10.1073/pnas.93.24.13780

Shaner NC, Lambert GG, Chammas A, Ni Y, Cranfill PJ, Baird MA, Sell BR, Allen JR, Day RN, Israelsson M, et al. 2013. A bright monomeric green fluorescent protein derived from Branchiostoma lanceolatum. Nat Methods 10: 407-409. doi:10.1038/nmeth.2413

Sherman F. 2002. Getting started with yeast. Methods Enzymol 350: 3-41. doi:10.1016/S0076-6879(02)50954-X

Sikorski RS, Hieter P. 1989. A system of shuttle vectors and yeast host strains designed for efficient manipulation of DNA in Saccharomyces cerevisiae. Genetics 122: 19-27.

Spira F, Mueller NS, Beck G, von Olshausen P, Beig J, WedlichSöldner R. 2012. Patchwork organization of the yeast plasma membrane into numerous coexisting domains. Nat Cell Biol 14: 640-648. doi:10.1038/ncb2487

Strádalová V, Stahlschmidt W, Grossmann G, Blazíková M, Rachel R, Tanner W, Malinsky J. 2009. Furrow-like invaginations of the yeast plasma membrane correspond to membrane compartment of Can1. J Cell Sci 122: 2887-2894. doi:10.1242/ jcs.051227

Sun Y, Miao Y, Yamane Y, Zhang C, Shokat KM, Takematsu H, Kozutsumi Y, Drubin DG. 2012. Orm protein phosphoregulation mediates transient sphingolipid biosynthesis response to heat stress via the Pkh-Ypk and Cdc55-PP2A pathways. Mol Biol Cell 23: 2388-2398. doi:10.1091/mbc.e12-03-0209

Tabuchi M, Audhya A, Parsons AB, Boone C, Emr SD. 2006. The phosphatidylinositol 4,5-biphosphate and TORC2 binding proteins $\operatorname{Slm} 1$ and $\operatorname{Slm} 2$ function in sphingolipid regulation. Mol Cell Biol 26: 5861-5875. doi:10.1128/MCB.02403-05

Tanigawa M, Kihara A, Terashima M, Takahara T, Maeda T. 2012. Sphingolipids regulate the yeast high-osmolarity glycerol response pathway. Mol Cell Biol 32: 2861-2870. doi:10.1128/MCB.06111-11

Tatebe H, Shiozaki K. 2017. Evolutionary conservation of the components in the TOR signaling pathways. Biomolecules 7: E77. doi:10.3390/biom7040077

Truman AW, Millson SH, Nuttall JM, King V, Mollapour M, Prodromou C, Pearl LH, Piper PW. 2006. Expressed in the yeast Saccharomyces cerevisiae, human ERK5 is a client of the Hsp90 chaperone that complements loss of the Slt2p (Mpk1p) cell integrity stress-activated protein kinase. Eukaryot Cell 5: 1914-1924. doi:10.1128/EC.00263-06

Vallejo MC, Mayinger P. 2015. Delayed turnover of unphosphorylated Ssk1 during carbon stress activates the yeast Hog 1 MAP kinase pathway. PLoS One 10: e0137199. doi:10.1371/journal. pone.0137199

Winkler A, Arkind C, Mattison CP, Burkholder A, Knoche K, Ota I. 2002. Heat stress activates the yeast high-osmolarity glycerol mitogen-activated protein kinase pathway, and protein tyrosine phosphatases are essential under heat stress. Eukaryot Cell 1: 163-173. doi:10.1128/EC.1.2.163-173.2002

Wullschleger S, Loewith R, Oppliger W, Hall MN. 2005. Molecular organization of target of rapamycin complex 2. J Biol Chem 280: 30697-30704. doi:10.1074/jbc.M505553200 


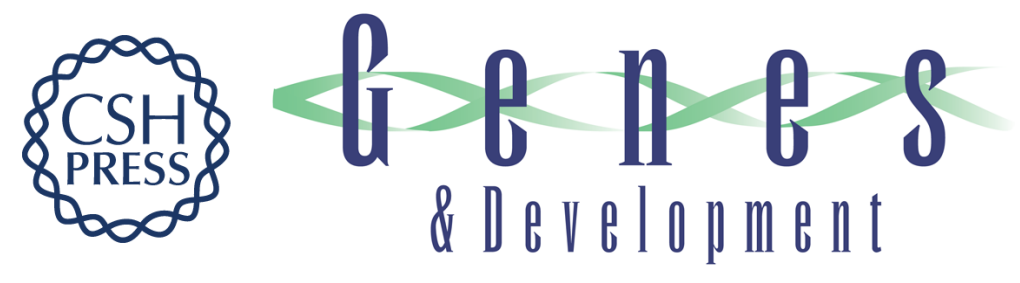

\section{Phosphorylation by the stress-activated MAPK Slt2 down-regulates the yeast TOR complex 2}

Kristin L. Leskoske, Françoise M. Roelants, Anita Emmerstorfer-Augustin, et al.

Genes Dev. 2018, 32: originally published online November 26, 2018

Access the most recent version at doi:10.1101/gad.318709.118

\section{Supplemental http://genesdev.cshlp.org/content/suppl/2018/11/23/gad.318709.118.DC1 Material}

References This article cites 80 articles, 49 of which can be accessed free at: http://genesdev.cshlp.org/content/32/23-24/1576.full.html\#ref-list-1

Creative This article, published in Genes \& Development, is available under a Creative Commons Commons License (Attribution 4.0 International), as described at License http://creativecommons.org/licenses/by/4.0/.

Email Alerting Receive free email alerts when new articles cite this article - sign up in the box at the top Service right corner of the article or click here.

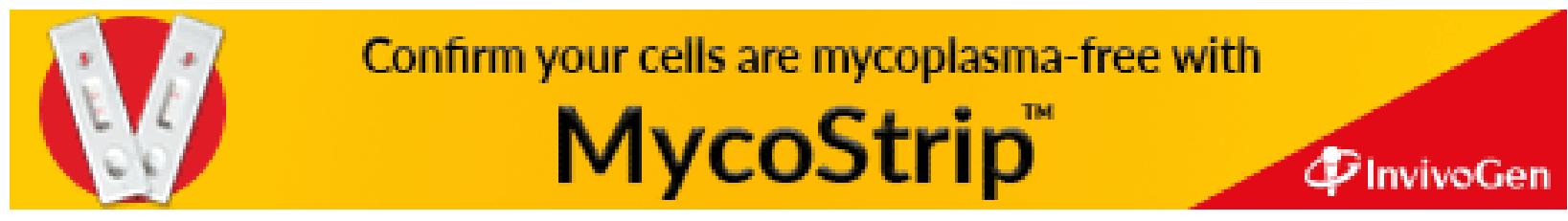

